\title{
Comparison of Multichannel Signal Deconvolution Algorithms in Airborne LiDAR Bathymetry Based on Wavelet Transform
}

Yue Song ( $\sim$ songyue199602@163.com )

Naval University of Engineering

Houpu Li

Naval University of Engineering

Guojun Zhai

Naval Institute of Marine Environment

Yan $\mathrm{He}$

Chinese Academy of Sciences

Shaofeng Bian

Naval University of Engineering

Wei Zhou

Guangxi Key Laboratory of Spatial Information and Geomatics

\section{Research Article}

Keywords: airborne LiDAR bathymetry, wavelet adaptive threshold denoising, signal deconvolution, multichannel waveform feature extraction

Posted Date: May 20th, 2021

DOl: https://doi.org/10.21203/rs.3.rs-533732/v1

License: (9) This work is licensed under a Creative Commons Attribution 4.0 International License. Read Full License

Version of Record: A version of this preprint was published at Scientific Reports on August 20th, 2021. See the published version at https://doi.org/10.1038/s41598-021-96551-w. 


\title{
Comparison of Multichannel Signal Deconvolution Algorithms in
}

\section{Airborne LiDAR Bathymetry Based on Wavelet Transform}

\author{
Yue Song ${ }^{1, *}$, Houpu Li ${ }^{1, *}$, Guojun Zhai ${ }^{2}$, Yan $\mathrm{He}^{3}$, Shaofeng Bian ${ }^{1}$ and Wei Zhou ${ }^{1,4}$ \\ 1. Department of Navigation Engineering, Naval University of Engineering, Wuhan 430033, \\ China; \\ 2. Naval Institute of Marine Environment, Tianjin 300061, China; \\ 3. Key Laboratory of Space Laser Communication and Detection Technology, Shanghai \\ Institute of Optics and Fine Mechanics, Chinese Academy of Sciences, Shanghai 201800, \\ China; \\ 4. Guangxi Key Laboratory of Spatial Information and Geomatics \\ * Correspondence Y : songyue199602@163.com; \\ * Correspondence $\mathrm{H}$ : lihoupu1985@126.com.
}

Airborne LiDAR bathymetry offers low cost and high mobility, making it an ideal option for shallow-water measurements. However, due to differences in the measurement environment and the laser emission channel, the received waveform is difficult to extract using a single algorithm. The choice of a suitable waveform processing method is thus extremely important to guarantee the accuracy of the bathymetric retrieval. In this work, we use a wavelet-denoising method to denoise the received waveform and then test four algorithms for denoised-waveform processing: Richardson-Lucy deconvolution (RLD), blind deconvolution (BD), Wiener filter deconvolution (WFD), and constrained least-squares filter deconvolution (RFD). The simulation database and the measured multichannel database are used to evaluate the algorithms, with the focus on improving their performance after the data-denoising preprocessing and their capability of extracting water depth. The results show that applying wavelet denoising before deconvolution improves the extraction accuracy. The four algorithms perform better for the shallow water orthogonal polarization channel (PMT2) than the shallow horizontal row polarization channel (PMT1). Of the four algorithms, RLD provides the best signal-detection rate, and RFD is the most robust. BD has low computational efficiency, and WFD performs poorly in deep water $(<25 \mathrm{~m})$.

Keywords: airborne LiDAR bathymetry; wavelet adaptive threshold denoising; signal deconvolution; multichannel waveform feature extraction

\section{Introduction}

Airborne LiDAR bathymetry (ALB) is an active remote-sensing technology and plays an important role in shallow-water topographic survey and measurement. It has the advantages of low operating cost, strong maneuverability, and high measurement accuracy and thus is widely used to update coastal topographic maps, for coastal construction, to monitor shallow waters, and in other fields [1-3]. ALB uses a strong penetrating green laser $(532 \mathrm{~nm})$ to scan waters less than $50 \mathrm{~m}$ deep. ALB emits blue and green laser beams and receives signals reflected various targets. The characteristic information is extracted from the difference in signal strength, and the technique is applied to measuring water depth and underwater topography and making low-quality 
classifications of the seabed [4-6]. A laser beam transmitted over a few hundred meters passes through the air and water to the seabed and then reflects back to the receiver. Along the way, a variety of noises perturb the signal, including noise internal to the receiver, atmospheric refraction, backscattering from water bodies, and diffuse reflection from the seabed. ALB is more complicated than terrestrial LiDAR measurements. Therefore, to process full-waveform ALB data, a crucial step is to improve the signal-to-noise ratio (SNR) of the data and accurately extract the echo signal [7].

In early airborne laser scanning (ALS) systems, only the transmitted signal and echo position signal were accepted; the complete echo signal was not retained to avoid storing redundant data. Pe'Eri proposed the use of two waveforms to make measurements in shallow waters. Near-infrared laser pulses are strongly reflected at the water surface, whereas a green laser beam penetrates the water much more easily, following which it reflects from the seabed [8]. Wong proposed using lowpass filters to remove noise from the signal and thereby extract the signal reflected from the seabed $[9,10]$. However, retaining only the transmitted signal and echo position signal may reduce target resolution, ranging accuracy, etc. At the same time, copious information about the water body (such as the turbidity of the water) cannot be extracted from the measurement. The study of Mallet shows the importance of using full-wave laser data to measure seafloor topography. He proposes that fullwave laser data are more conducive to extracting target position and for research on backscattering from water bodies [3,11]. Characterization of full-wave laser data is thus important to increase our understanding of laser beam propagation. At present, processing algorithms for full-wave green laser data can be divided into the following three categories:

- Target-detection method: Depending on the original waveform, the target-detection method extracts the reflected laser energy time by identifying the mutation point of the continuous signal reflection energy. Common target-detection methods include peak detection (PD), firstorder derivative (FD), and the average square function (ASDF) [12-14]. The algorithm is fast but is susceptible to gross errors and noise. It is generally used to extract the initial value and extract the reflection time point after data preprocessing.

- Waveform decomposition method: The waveform decomposition method treats the laser waveform as a superposition of multiple mathematical functions and uses a combination of multiple mathematical functions to fit the waveform, thereby restoring the true original signal. Commonly used fitting functions include Gaussians, logarithmic normal distribution functions, and quadrilateral functions [15-17]. Gaussian functions are widely used for fitting; for example, Gaussian fitting based on the nonlinear least-squares method [18], Kai Guo based on Gaussian fitting [19], and Gaussian half-wave width to decompose the waveform and extract the effective part of the data to suppress noise, which improves somewhat the problem of Gaussian overfitting. Wang [14] compared quadrilateral fitting with mean variance fitting, but the waveform fitting algorithm has difficulty solving the problem of signal overlap, such as the overlap of a surface echo and a bottom echo. The backscattering of the water body is an asymmetric waveform, so it is difficult to fit the Gaussian function.

- Deconvolution algorithm: The deconvolution algorithm regards the received waveform as the convolution of the laser emission pulse and the target cross section. Commonly used deconvolution algorithms include the Wiener filter deconvolution [20], Richardson-Lucy deconvolution (RLD) [21], blind deconvolution (BD) [14], and B-spline deconvolution [22]. However, the deconvolution algorithm is more affected by noise, which can lead to 
misjudgment of reflected echoes, and the data processing results are easily affected by ringing.

The three types of data processing methods for green lasers show that target detection is relatively simple and does not depend on the laser propagation process; however, the accuracy is poor. The waveform decomposition method is an approximate fitting to the full waveform data, and most waveform fitting functions are symmetrical functions. The waveform decomposition method does not consider the backscattering of the water body, which makes for unsatisfactory fitting. However, the deconvolution algorithm starts from the laser propagation and waveform formation process and uses the inverse transformation of the waveform formation to restore a more realistic target cross section. If noise can be removed, the deconvolution algorithm is an ideal method for laser echo processing. At present, wavelet theory is widely used in digital signal processing [23], and the denoising effect is relatively ideal.

This paper first establishes a water-depth radar model (Wa-LiD) that is closer to reality according to the airborne laser propagation and formation process and then applies wavelet denoising to the simulation results to find the best denoising parameters. Next, the denoised data are deconvoluted, the advantages and disadvantages of various algorithms are compared, and the influence of the parameters on the echo received by the laser is analyzed. Finally, the results of applying the algorithm to the simulation data and to measured data are comprehensively evaluated to determine the optimal deconvolution method. This lays the foundation for extracting water depth by airborne laser.

\section{Materials and Methods}

\subsection{Simulated dataset and acquisition dataset}

\subsubsection{Simulated dataset}

The Wa-LiD model is simulated by applying the reflection and refraction of different wavelengths in a complex water environment to form a reflected wave. The result of the simulation is closer to the actual ALB waveform and can amplify the influence of various factors on the waveform, which facilitates the comparison of various parameters in waveform denoising and deconvolution. In addition, during the actual flight measurement, since no noise-free waveform data can be obtained, the accuracy of the algorithm is often evaluated by using statistically relative truth values, and the simulation data can produce completely-noise-free simulation truth values, which promotes the accuracy of the algorithm. Evaluation, therefore, is particularly important to obtain laser simulation results that are closer to reality. The wavelength range of the Wa-LiD model [24] is 300-1500 $\mathrm{nm}$. The laser used by the ALB system produces long-wave $1064 \mathrm{~nm}$ infrared light and $532 \mathrm{~nm}$ blue-green light. Infrared light cannot penetrate the water body and is reflected from its surface. Blue-green light penetrates the water body and reflects from the bottom. Therefore, the model uses $532 \mathrm{~nm}$ green light, which has strong penetrability. The echo waveform of the ALB system can be expressed as the superposition of multiple echoes:

$$
p_{r}(\mathrm{t})=p_{s}(\mathrm{t})+p_{c}(\mathrm{t})+p_{b}(\mathrm{t})+p_{N}(\mathrm{t}),
$$

where $p_{s}(\mathrm{t})$ is reflection from the water surface, $p_{c}(\mathrm{t})$ is reflection from the water column scattering, $p_{b}(\mathrm{t})$ is reflection from the bottom, and $p_{N}(\mathrm{t})$ is the ALB system noise. The laser radar emission pulse can be expressed by a Gaussian function [20]:

$$
w_{t}\left(t_{x}\right)=\frac{2}{T_{0}} \sqrt{\frac{\ln 2}{\pi}} \exp \left(-4 i n 2 \frac{\left(t-t_{x}\right)^{2}}{T_{0}^{2}}\right),
$$

where $T_{0}$ is the full width at half maximum of the transmitted pulse, and $t_{x}$ is the round-trip time for the transmitted pulse to reach the target. 
(1) Water surface reflection model

After the laser pulse is emitted from the transmitter, it passes through the air and reflects from the water surface. The reflected energy received by the ALB detector can be expressed as the convolution of the transmitted pulse and the water surface echo energy $p_{s}$ :

$$
p_{s}(t)=w\left(t_{s}\right) \otimes P_{s},
$$

where $\otimes$ indicates the convolution and $p_{s}$ is

$$
p_{S}=\frac{P_{e} T_{\mathrm{atm}}^{2} A_{R} \eta_{e} \eta_{R} L_{S} \cos ^{2}\left(i_{\text {laser }}\right)}{\pi H^{2}}
$$

where $P_{e}=\frac{E_{0}}{T_{0}}$ is the laser emission power, $E_{0}$ is the laser emission energy, $T_{\mathrm{atm}}^{2}$ is the atmospheric double-pass loss factor, $A_{R}$ is the receiving area of the receiver, $\eta_{e}$ is the optical emission efficiency of the laser transmitter, $\eta_{R}$ is the optical receiving efficiency of the receiver, $i_{\text {laser }}$ is the incident angle of the laser beam with respect to the normal of the water surface, $H$ is the sensor height, and $L_{S}$ is the laser the transmission loss in traveling to the water surface. $L_{S}$ is expressed as

$$
L_{S}=\frac{k_{d}}{\pi}+\frac{k_{s} e^{\left(-\tan i_{\text {laser }} / r\right)^{2}} \alpha_{\mathrm{BRDF}} F_{r}}{\pi r^{2} \cos ^{6}\left(i_{\text {laser }}\right)},
$$

where $k_{d}$ is the diffuse reflection coefficient of the water surface, and $k_{s}$ is the specular reflection coefficient. The relationship between $k_{d}$ and $k_{s}$ is

$$
k_{d}=1-k_{s}
$$

where $r$ is the water surface roughness, $\alpha_{\mathrm{BRDF}}$ is the geometric attenuation coefficient of the water surface, and $F_{r}$ is the function that describes the Fresnel reflection of light from each microfacet.

(2) Water reflection model

The echo reflection signal from depth $D$ received by the ALB system receiver can be expressed as the convolution of the transmitted pulse and the instantaneous echo energy at $D$, which is expressed as

$$
p_{c}(t)=w\left(t_{c}\right) \otimes p_{c}(D) .
$$

The instantaneous echo energy at $D_{i}$ can be expressed as

$$
p_{c}\left(D_{i}\right)=\frac{P_{e} T_{\mathrm{atm}}^{2} A_{R} \eta_{e} \eta_{R} F\left(1-L_{S}\right)^{2} \beta(\varnothing) \exp \left(\frac{-2 k D_{i}}{\cos r_{\text {laser }}}\right)}{\left(\frac{n_{W} H+D_{i}}{\cos i_{\text {laser }}}\right)^{2}}
$$

where $F$ is the field-angle loss factor, $\beta(\varnothing)$ is the body scattering function, $n_{w}$ is the water refractive index, $H$ is the sensor altitude, $r_{\text {laser }}$ is the angle of refraction of the laser at the water surface, $D_{i}$ is the depth reached by the laser beam in the water, and $k$ is the diffuse attenuation coefficient of the water.

(3) Underwater reflection model

The echo energy at the bottom $Z$ received by the ALB system receiver can be expressed as the convolution of the echo at $Z$ and the transmitted pulse, with the echo energy at $Z$ expressed as

$$
p_{b}\left(D_{i}\right)=\frac{P_{e} T_{\mathrm{atm}}^{2} A_{R} \eta_{e} \eta_{R} F\left(1-L_{S}\right)^{2} R_{b} \exp \left(\frac{-2 k Z}{\cos r_{\text {laser }}}\right)}{\pi\left(\frac{n_{W} H+Z}{\cos i_{\text {laser }}}\right)^{2}},
$$

where $R_{b}$ is the bottom reflectance.

(4) ALB system noise model 
The ALB system noise mainly includes the system background noise and the internal noise of the instrument. The internal noise of the instrument can be expressed as a normal distribution with $\mu=0, \sigma=1$, where

$$
\sigma_{N}(\mathrm{t})=\frac{1}{\sqrt{2 \pi} \sigma} e^{\frac{-(t-\mu)^{2}}{2 \sigma^{2}}}
$$

The background noise can be expressed as $p_{B N}=p_{b a} * \sigma_{g}$, where $p_{b a}$ can be expressed as

$$
p_{b a}=I_{s} A_{R} T_{a t m}^{2}\left(1-\gamma_{r}^{2}\right) \frac{\pi \theta^{2}}{4} \Delta_{\lambda} \eta_{R},
$$

where $I_{S}$ is the intensity of the solar reflection from the water body, and $\Delta_{\lambda}$ is the bandwidth of the optical filter of the receiver.

We use the above Wa-LiD model to simulate two types of echo waveform data with noise (Figure 1b) and without noise (Figure 1a).

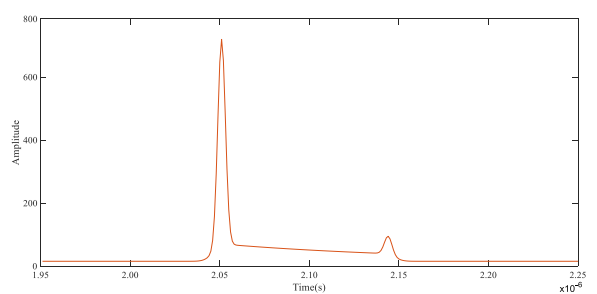

(a)

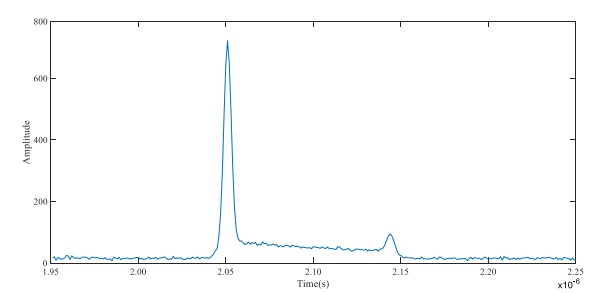

(b)

Figure 1. ALB simulation waveform. (a) Noise-free simulation waveform, (b) Simulation waveform with noise. $E_{0}=20 \mathrm{~mJ}, T_{0}=5 \mathrm{~ns}, i_{\text {laser }}=0.3, T_{\mathrm{atm}}^{2}=0.9, A_{R}=0.025 \mathrm{~m}^{2}, \eta_{e}=$ $0.9, \eta_{R}=0.5, k_{d}=0.1, k_{s}=0.9, F_{r}=0.2, Z=10 \mathrm{~m}, \beta(\varnothing)=0.0014$.

\subsubsection{Acquisition dataset}

The measured data are acquired by the airborne laser ALB system (Mapper5000) developed by the Shanghai Institute of Optics and Fine Mechanics, the Chinese Academy of Sciences on Ganquan Island, Hainan Province, China. The Mapper5000 system uses a four-channel high-speed waveform acquisition card for wavelengths of 1550, 1064, and $532 \mathrm{~nm}$ for echo collection. The sampling rate of each channel reaches $1 \mathrm{GSa} / \mathrm{s}$, and the digital resolution is $10 \mathrm{bits}$. It includes three green channels [532 nm, photomultiplier tube (PMT)] and one near-infrared channel [1064 nm, avalanche photodiode (APD)]. The three green channels are the shallow horizontal row polarization channel (PMT1), the shallow-water orthogonal polarization channel (PMT2), and the deep-water channel (PMT3). This work makes a comparative analysis of the measured data from PMT1 and PMT2 and uses the seabed echo signal extracted from the PMT3 data and the water surface reflection signal received by the APD as the reference true value of the actual measurement for evaluating the performance of the algorithm for data processing. The red wire frame in Figure 2 shows the measured area of the experimental data. 


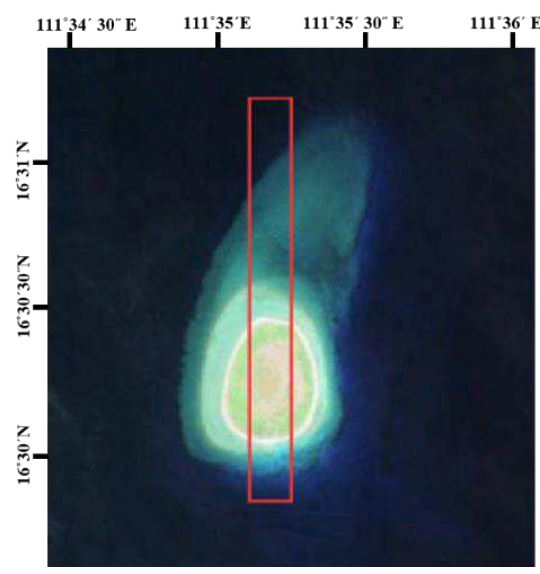

Figure 2. Study area, the red wire frame indicates the Mapper5000 aerial survey area.

\subsection{Methods}

In processing the airborne laser waveform data, suppressing the noise of the waveform data is an important step in preprocessing the waveform. The basic idea of the convolution algorithm is to regard the received waveform as the convolution of the laser emission pulse $w\left(t_{s}\right)$ and the target cross section $\mathrm{C}(\mathrm{t})$ plus noise, then the received waveform can be expressed as

$$
p_{r}(\mathrm{t})=w_{t}(t) \otimes \mathrm{C}(\mathrm{t})+\mathrm{n}(\mathrm{t}),
$$

where $n(t)$ is the noise on the received waveform. If the influence of the noise on the received waveform is reduced as much as possible before the waveform is deconvolved, the accuracy of the waveform after deconvolution can be greatly improved because, when $n(t)$ is small enough, the received waveform can be regarded simply as a laser emission pulse convolution of $w\left(t_{s}\right)$ with the target cross section $\mathrm{C}(\mathrm{t})$. The waveform after deconvolution is closer to the target cross section, so the seabed terrain will be more accurately extracted. Many ways exist to denoise the waveform, for example, using filters to remove high-frequency noise, or setting thresholds for the half width and amplitude of the received waveform [25]. However, the soft threshold wavelet method produces better denoising of the deconvoluted waveform. Since the wavelet transform is a linear transform, the echo signal after orthogonal transform removes the correlation between the original signals to the maximum extent. The information from each component signal is retained, which provides a basis for the subsequent deconvolution to separate the cross section.

\subsubsection{Workflow}

The experiment in this article is divided into three steps. In the first step, the simulation results are used for the denoising experiment, and the simulation parameters are adjusted to maximize the approximation of the real received echo. The three main parameters in wavelet denoising (wavelet base, decomposition level, and denoising threshold) are compared and analyzed to obtain the optimal denoising parameters and lay the foundation for the next deconvolution operation.

In the second step, the denoising data undergo four deconvolution operations, and the comparison with the direct deconvolution data proves the necessity of denoising experiments. Simulation results are used to explore how the parameters affect the deconvolution algorithm. In the third step, the PMI1 and PMI2 channel data are used to evaluate the algorithm, where the surface position extracted from the APD data and the bottom position extracted from PMI3 are used as the 
true values of the measured data. Figure 3 shows a flow chart describing the experimental process.

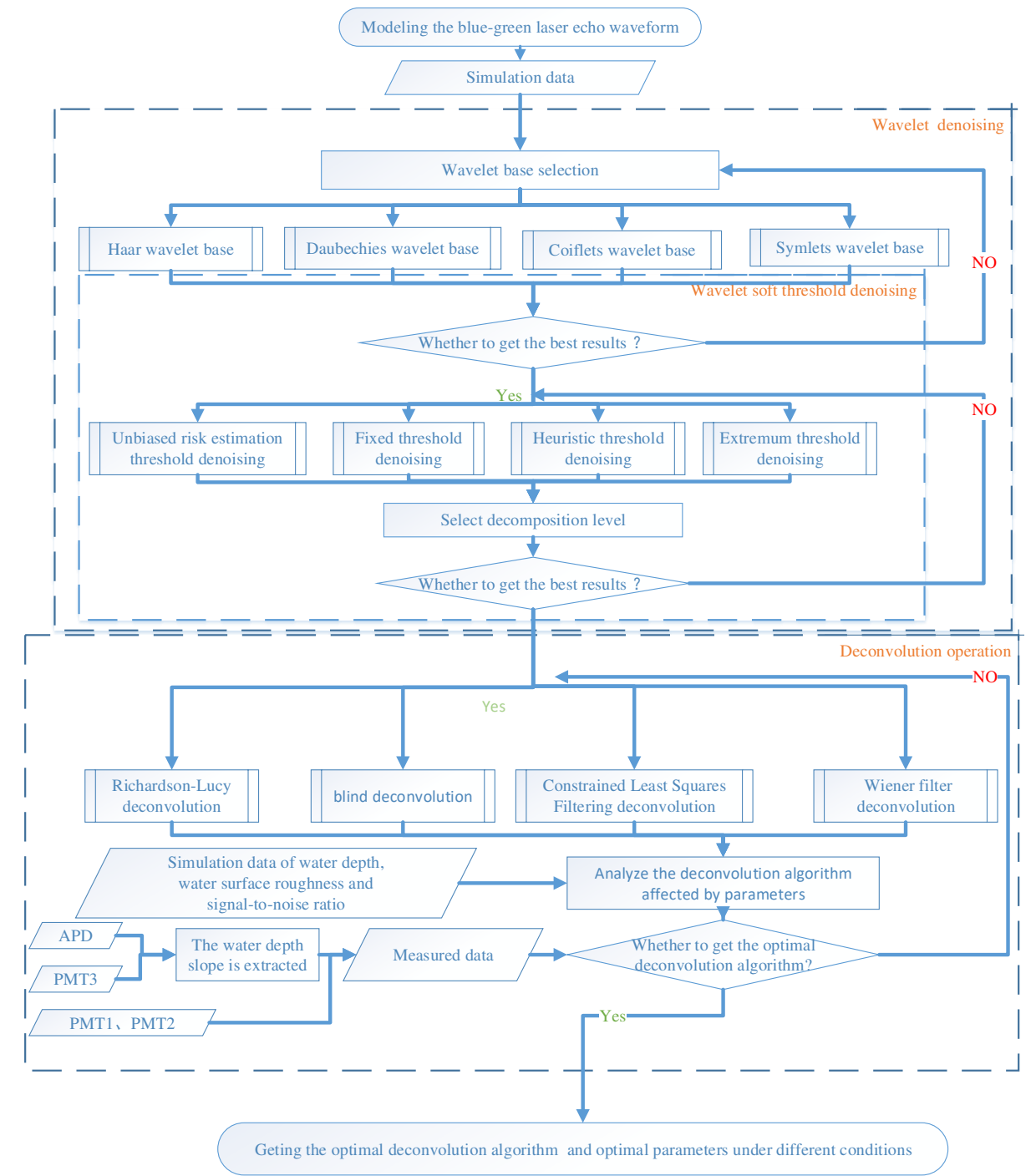

Figure 3. Flow chart of experimental process.

\subsubsection{Wavelet transform denoising algorithm}

The wavelet transform is divided into high-frequency coefficients and low-frequency coefficients. The high-frequency coefficients reflect the noise and sudden changes in the signal, and the low-frequency coefficients reflect the overall trend and correlation of the signal [26,27]. Therefore, the high-frequency signal after wavelet transformation is subjected to wavelet threshold processing. The threshold processing function can be divided into a hard threshold processing method and a soft threshold processing method. The latter can be expressed as

$$
w_{\lambda}= \begin{cases}{[\operatorname{sign}(w)](|w|-\lambda)} & |w| \geq \lambda \\ 0 & |w|<\lambda,\end{cases}
$$

where $\mathrm{w}$ is the wavelet decomposition coefficient, $\lambda$ is the threshold, and sign(w) is the sign of w. The soft threshold processing serves to "shrink" the wavelet coefficients so that the input and output curves are continuous. Many researchers find that denoising with the soft threshold function provides better results than denoising with the hard threshold function [28], so we use herein the soft-threshold denoising method. The wavelet denoising threshold is mainly divided into four parts:

(1) Unbiased risk-estimation threshold 
The original signal is arranged by taking the absolute value from small to large signals, squaring each, and using the square root of element $k$ as the threshold. The risk expression generated by the threshold is

$$
\operatorname{Risk}(k)=\left[N-2 k+\sum_{i=1}^{k} f(i)+(N-k) f(N-k)\right] / N,
$$

where $N$ is the total number of data, and $k$ is element $k$. Therefore, the element with the least risk serves as the unbiased risk-estimation threshold.

(2) Fixed threshold

(3) Heursure threshold

$$
\lambda=\sqrt{2 \ln (N)} .
$$

$$
\begin{gathered}
\text { crit }=\sqrt{\frac{1}{N}\left(\frac{\ln (N)}{\ln (2)}\right)}, \\
\text { eta }=\left[\sum_{j=1}^{N}\left|S_{j}\right|^{2}-N\right] / n .
\end{gathered}
$$

When eta $<$ crit, the fixed threshold is used; otherwise, the unbiased risk threshold is used.

(4) Extreme value threshold

$$
\lambda= \begin{cases}0.3936+0.1829\left(\frac{\ln N}{\ln 2}\right), & N>32 \\ 0, & N<32 .\end{cases}
$$

\subsubsection{Waveform deconvolution algorithm}

(1) Wiener filter deconvolution

WFD assumes that the signal and noise are independent, and the Wiener filter $F(t)$ is used to reduce the gap between the actual target cross section $\mathrm{c}(\mathrm{t})$ and the evaluated target cross section $\bar{c}(t)$ [29].The Wiener filter $F(t)$ can be expressed in the frequency domain as

$$
F(f)=\frac{\left|W_{t}(f)\right|^{2}}{\left|W_{t}(f)\right|^{2}+K}
$$

where $K$ is a parameter related to noise whose value is to be determined through multiple experiments before using Wiener filtering. The time-domain estimate of the final cross section is

$$
\bar{p}(t)=F F T^{-1}\left(\frac{P_{r}(f) F(f)}{W_{t}(f)}\right) .
$$

(2) Constrained least square filter deconvolution

RFD is generally used for image restoration. WFD requires that the power spectrum of the undegraded image and noise be known. These two power spectra are usually difficult to estimate, while the RFD requires only the variance and mean of the noise [30]. These parameters can be calculated from the given received waveform, which is the advantage of constrained least square filtering [31]. The core problem of RFD is to solve the problem of the sensitivity to noise of the degradation function. "Degenerate function" is a term used in image processing. In waveform signal processing, a degenerate function may be understood as the convolution of the transmitted signal and the target cross section. To reduce the sensitivity to noise of the convolution, a minimum criterion function $\mathrm{C}$ with constraints is established,

$$
\mathrm{C}=\sum_{0}^{M-1}\left[\nabla^{2} w(t)\right]^{2},
$$

where $\nabla^{2}$ is the Laplacian operator, which is used to express the smoothness of the waveform.

The constraints are

$$
\left\|p_{\mathrm{r}}(\mathrm{t})-\mathrm{D} \bar{p}(t)\right\|^{2}=\left\|p_{N}(\mathrm{t})\right\|^{2} .
$$


(3) Richardson-Lucy deconvolution [32]

RLD uses an iterative process to deconvolute the signal, which restores the likelihood of the signal by using an expectation maximization algorithm in the time domain. Iteration $i$ is expressed as

$$
\bar{c}^{i+1}(t)=\bar{c}^{i}(t)\left[w_{t}(t) \otimes \frac{w_{r}(t)}{\bar{c}^{i}(t) \otimes w_{t}(t)}\right],
$$

where $\bar{c}^{i+1}(t)(\mathrm{t})$ and $\bar{c}^{i}(t)$ represent iteration $i+1$ and $i$, respectively.

(4) Blind deconvolution

$\mathrm{BD}$ is similar to RLD, except that the received signal is iterated under the assumption that the point spread function is not known, and the point spread function (transmitted signal) and the horizontal cross-sectional waveform are estimated at the same time, so the gap between the original and the estimated cross sections is reduced after multiple iterations [33]. Iteration $k-1$ yields the cross section $c_{k-1}, w_{t_{k}}$ is obtained by using the Richardson-Lucy formula, and $c_{k}$ is obtained from $w_{t_{k}}$. Repeated iterations give finally $\bar{c}$ and $\bar{w}_{t}$. The iteration formula is as follows:

$$
\begin{array}{r}
{\overline{w_{t}}}_{i+1}^{k}(t)=\bar{w}_{t_{i}}{ }^{k}(t)\left[\bar{c}^{k-1}(t) \otimes \frac{w_{r}(t)}{\bar{w}_{t_{i}}^{k}(t) \otimes \bar{c}^{k-1}(t)}\right], \\
\bar{c}_{i+1}^{k}(t)=\bar{c}_{i}{ }^{k}(t)\left[\bar{w}_{t}{ }^{k-1}(t) \otimes \frac{w_{r}(t)}{\bar{w}_{t}^{k}(t) \otimes \bar{c}^{k}(t)}\right] .
\end{array}
$$

\section{Results}

3.1 Experimental comparison of wavelet denoising parameters

The quality of the wavelet-transform denoising algorithm depends mainly on the choice of wavelet base, the decomposition level, and the threshold. These three parameters are compared and verified by simulation results to provide a good data basis for the deconvolution algorithm.

\subsubsection{Experiment to compare wavelet basis}

We use herein four common wavelet bases for experiments, namely, Haar, Daubechies, Coiflets, and Symlets wavelets (abbreviated haar, db, coif, and sym wavelets). The choice of wavelet base mainly considers orthogonality, tight support, symmetry, vanishing distance, and regularity. Orthogonality means that the inner product of every pair of wavelet bases is zero, so all four are orthogonal functions. Supportability means that, if the function can only take a value near zero, the range of values is called a "compact support set." Symmetry refers to whether the wavelet basis function is symmetric, and vanishing distance ensures that as many wavelet coefficients as possible are zero or as small as possible. The nonzero wavelet coefficients help to eliminate noise, and regularity refers to the smoothness of the wavelet basis function. Taking a water depth of $10 \mathrm{~m}$ as an example, we compare four wavelet denoising effects under different SNRs (Figure 3). The SNR is a parameter that reflects the intensity of noise on the signal. It is expressed as

$$
\mathrm{SNR}=10 \log \left(\frac{P_{s}}{P_{v}}\right),
$$

where $P_{S}$ is the average power of the noise-free signal, and $P_{v}$ is the average power of the noise.

Table 1. Wavelet basis function performance.

\begin{tabular}{ccccc}
\hline Wavelet function & Haar & Daubechies & Coiflets & Symlets \\
\hline Abbreviation & haar & $\mathrm{db}$ & coif & sym \\
Orthogonality & Yes & Yes & Yes & Yes \\
\hline
\end{tabular}




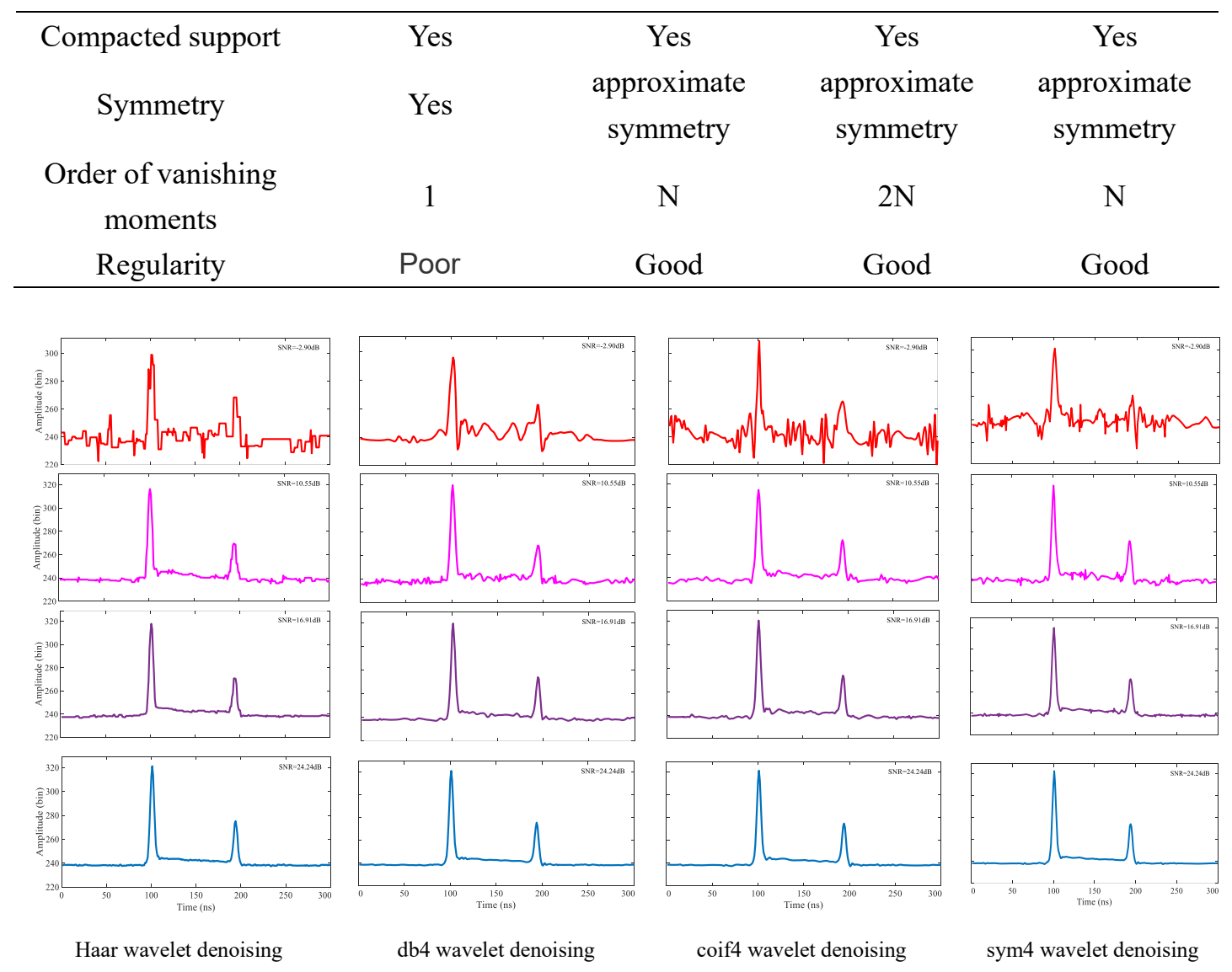

Figure 4. Effect of different wavelet basis functions on waveform denoising.

Table 1 shows that the Haar wavelet is more symmetric than other wavelet basis functions and has a smaller range of tightly supported sets, but it is less regular than the other three wavelet bases. In the experiment, we used the fourth-order Daubechie wavelet base (db4) and the fourth-order Coiflets wavelet base (coif4). Figure 4 shows that, in the case of a low SNR and after Haar wavelet denoising, the waveform is rectangular, and other wavelet-basis denoising is relatively smooth. However, judging from the denoising of the four basis functions in Figure 4, the four wavelet basis functions all show better denoising effects when the signal-to-noise ratio is high. With decreasing $\mathrm{SNR}$, the $\mathrm{db} 4$ and Haar wavelet bases produce better denoising than the coif and sym4 wavelet bases. For the denoising effect of the red waveform data in the fourth row of Figure 4, the RNS is $2.90 \mathrm{~dB}$, and the data after denoising by the $\mathrm{db} 4$ and haar wavelets can still identify clearer reflections from the water surface and from the bottom. However, considering that the Haar wavelet is a rectangular wave and the original waveform data are Gaussian-like waveforms, to avoid introducing more errors and to accurately extract the reflection position of the bottom echo, we use herein the db4 wavelet basis to denoise the ALB laser echo.

3.1.2 Selection of wavelet denoising threshold and decomposition layer number

This section uses the $\mathrm{db} 4$ wavelet basis function to evaluate the denoising effect of the four soft thresholds. The data are acquired from a water depth of $10 \mathrm{~m}$ as an example, and SNR $=16.91 \mathrm{~dB}$. 


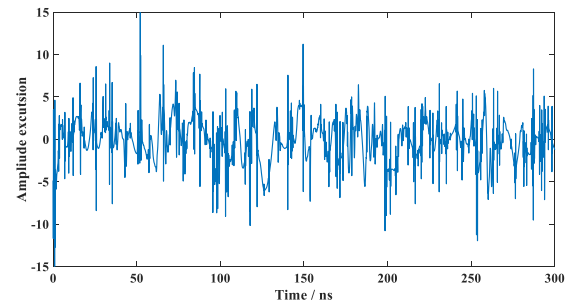

(a)

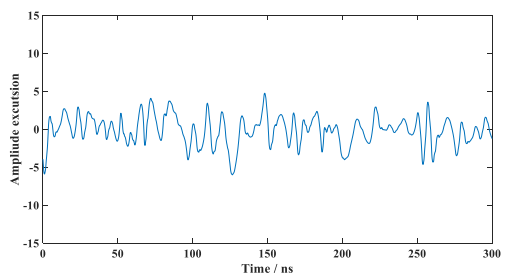

(c)

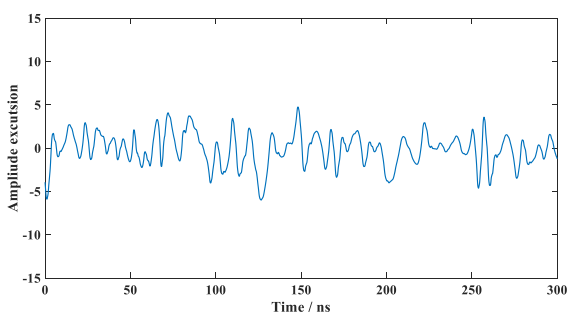

(b)

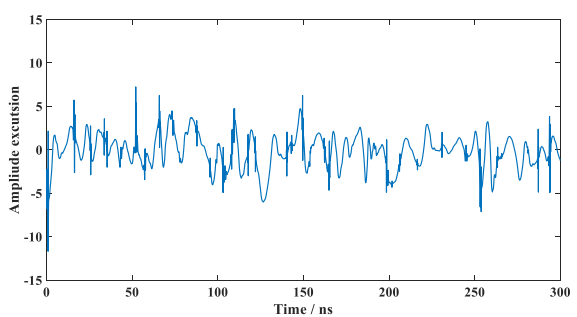

(d)

Figure 5. Denoising deviation diagram of various thresholds: (a) unbiased risk estimation threshold denoising, (b) fixed threshold denoising, (c) heuristic threshold denoising, (d) extreme value threshold denoising.

To clarify the comparison after denoising, Figure 5 shows the difference between the four denoising methods and the original noise-free signal after six-layer wavelet decomposition and denoising. The unbiased risk estimation threshold denoising performs the worst, followed by the extreme threshold denoising; the best denoising is provided by heuristic threshold denoising and fixed threshold denoising, which provide equivalent denoising.

The wavelet decomposition level is related to the data capacity and is one of the main parameters that affect denoising. Therefore, we obtain the optimal number of denoising decomposition layers of ALB by increasing the number of decomposition layers one by one. The denoising accuracy is quantified by the root mean square error (RMSE):

$$
\text { RMSE }=\sqrt{\frac{1}{N} \sum_{i=1}^{N}\left(f\left(t_{i}\right)-y_{i}\right)^{2}},
$$

where $N$ is the number of signal samples, $f\left(t_{i}\right)$ is the denoising signal, and $y_{i}$ is the original noisefree signal.

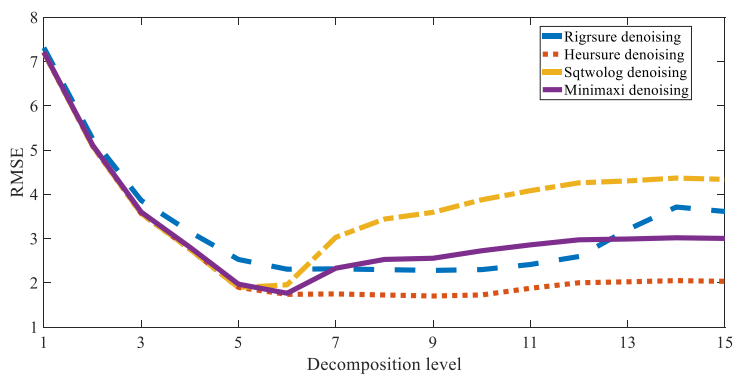

Figure 6. RMSE of denoising accuracy as a function of decomposition level to compare accuracies of wavelet threshold denoising decomposition levels.

Figure 6 shows the RMSE for the first to fifteenth order decomposition levels of the four threshold wavelet denoising algorithms. These results show that the accuracy of the four soft 
threshold wavelet denoising methods has improved rapidly in the first six-order decomposition. Heuristic threshold denoising provides the highest accuracy (i.e., lowest RMSE) in the sixth order, which tends to remain stable at higher orders. The accuracy of the other denoising algorithms worsens at higher orders. To summarize, for soft threshold wavelet denoising, heuristic threshold denoising provides the best echo signal denoising, and six decomposition layers are optimal for denoising.

\subsection{Experiment to compare deconvolution algorithms}

To explore how the wavelet denoising algorithm affects the deconvolution algorithm, Figure 7 shows how the deconvolution operation affects the data before and after wavelet denoising and compares the effect of data deconvolution.

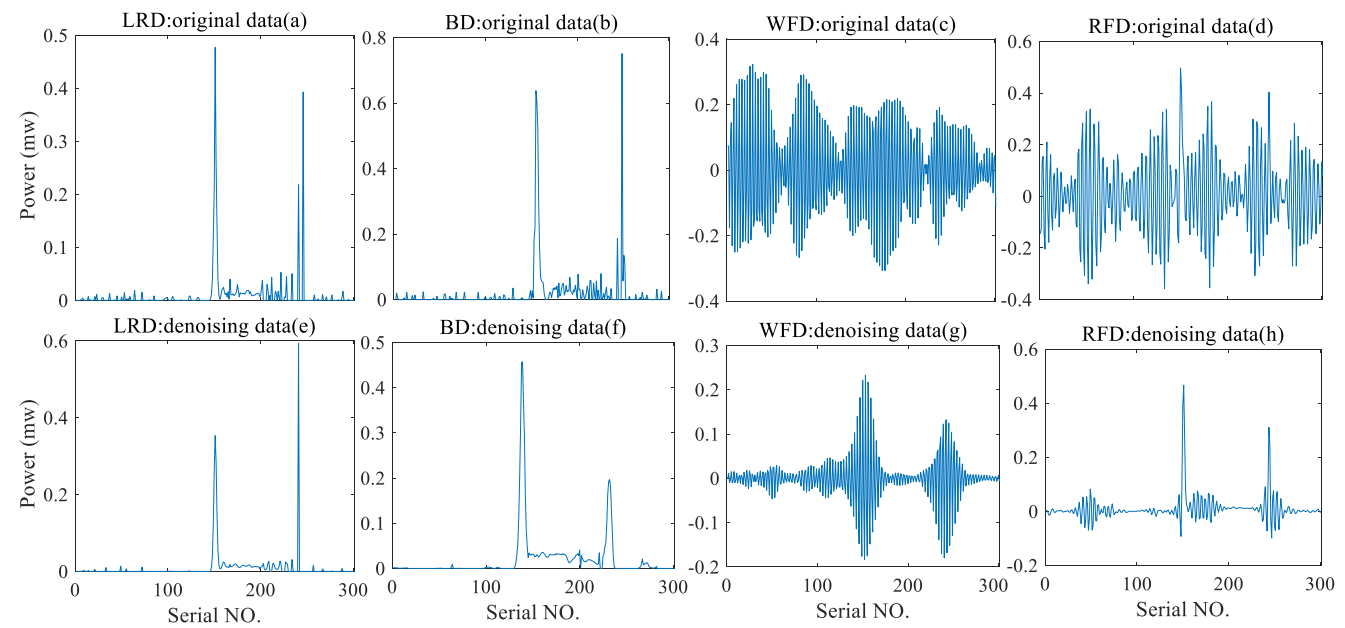

Figure 7. Comparison of deconvolution algorithms.

Figure 7 shows that the data after wavelet denoising produces a better deconvolution. Figures $7(\mathrm{a}), 7(\mathrm{~b}), 7(\mathrm{e})$, and 7(f) show that the waveform of the denoising data becomes smooth after deconvolution, and the reflected echo is retained. Since the deconvolution operation can improve the data resolution, it also amplifies the impact of noise on the data. In Figure 7(a), significant noise interference appears around the bottom reflection echo. However, wavelet denoising removes the interference, thereby improving the strength of the bottom reflection. The effect of wavelet denoising is clearer with the WFD and RFD algorithms. Before wavelet denoising, the noise of the WFD and RFD algorithm solution results is completely fused with the target reflection signal, and effective information cannot be extracted. After wavelet denoising, the noise is suppressed, so the target reflection becomes more obvious.

\section{Discussion}

\subsection{Comparative analysis of simulation data}

To further analyze how processing affects each deconvolution algorithm, we add the following algorithm-evaluation indicators to evaluate how processing affects the deconvolution algorithm:

(1) Root mean square error of water depth slope distance

Since the deconvolution algorithm restores the cross-sectional shape of the target, evaluating the accuracy of the water-depth data better reflects the quality of the algorithm. The RMSE of the water-depth slope distance is expressed as: 


$$
R M S E_{D}=\sqrt{\frac{\sum_{i}^{N}\left(Z_{i}^{\prime}-Z_{i}\right)^{2}}{N}}
$$

where $Z_{i}^{\prime}$ and $Z_{i}$ are the estimated aquatic slope distance and the true water depth slope distance, respectively, and $N$ is the number of samples.

\section{(2) Correlation coefficient}

We calculate the correlation coefficient (CORR) between the deconvolution result and the true reflected echo:

$$
\operatorname{CORR}=\frac{\sum_{i}^{N}\left(M_{i}^{\prime}-\bar{M}_{i}^{\prime}\right)\left(M_{i}-\bar{M}\right)}{\sqrt{\sum_{i}^{N}\left(M_{i}^{\prime}-\bar{M}_{i}^{\prime}\right)^{2} \sum_{i}^{N}\left(M_{i}-\bar{M}\right)^{2}}},
$$

where $M_{i}^{\prime}$ is the waveform processed by the algorithm, and $M_{i}$ is the reflection from the bottom. The closer the correlation coefficient is to unity, the stronger the correlation is between the two.

(3) Goodness of fit

$$
R^{2}=1-\frac{\sum_{i}^{N}\left(Z_{i}^{\prime}-Z_{i}\right)^{2}}{\sum_{i}^{N}\left(Z_{i}-\bar{Z}\right)^{2}}
$$

The goodness of fit $R^{2}$ describes the degree of fit between the extracted water depth and the actual water depth. The fitting improves as $R^{2}$ approaches unity.

(4) The time $T$ required to evaluate the computational efficiency of the algorithm.

Table 2. Comparison of performance of deconvolution algorithms.

\begin{tabular}{ccccc}
\hline Evaluation parameter & RLD & BD & WFD & RFD \\
\hline RMSE $(m)$ & 0.1015 & 0.4220 & 0.6059 & 0.0435 \\
CORR & 0.8226 & 0.8791 & 0.8262 & 0.8516 \\
R2 & 0.9910 & 0.8837 & 0.9663 & 0.9664 \\
T(s) & 0.8194 & 0.9249 & 0.3681 & 0.4345 \\
\hline
\end{tabular}

Table 2 lists the results of the deconvolution of 100 sets of wavelet denoising and denoising simulation data. The RFD algorithm is the most accurate and requires the shortest calculation time. The accuracy of the WFD algorithm is relatively poor, but over the course of the experiment, it becomes more stable than RFD. The RFD algorithm needs to repeatedly adjust the convolution parameters to achieve the optimal effect. The RLD algorithm deconvolutes the waveform through iteration, which takes a relatively long time. However, unlike the RLD algorithm, the BD algorithm assumes that the point spread function is not known, so it must be estimated before running the BD algorithm. This increases the calculation time and reduces the accuracy.

Based on the deconvolution performance of each algorithm, we further explore how changes in the main parameters of the laser echo affect the algorithms (see Figures 8-10). 


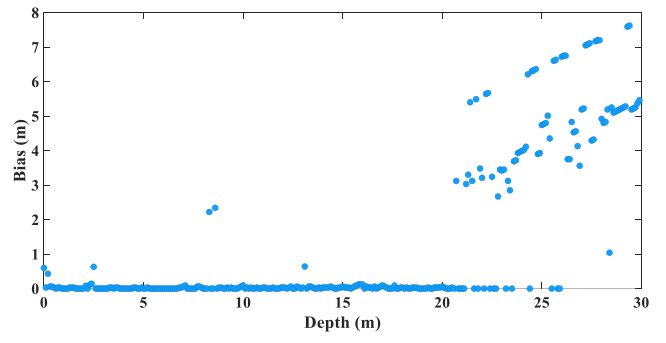

(a)

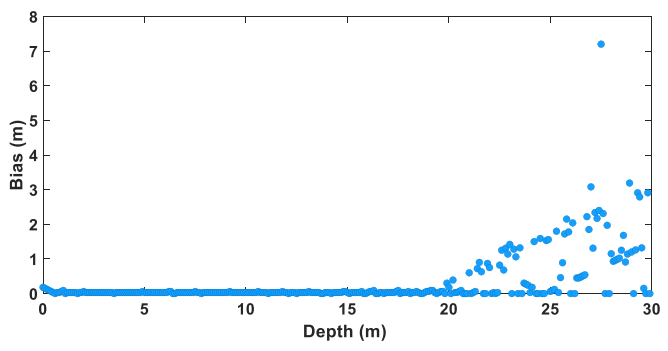

(c)

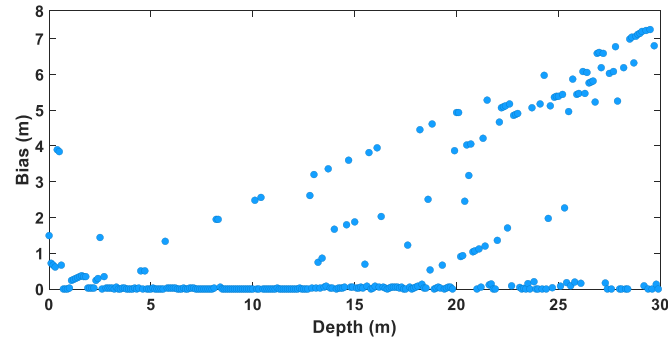

(b)

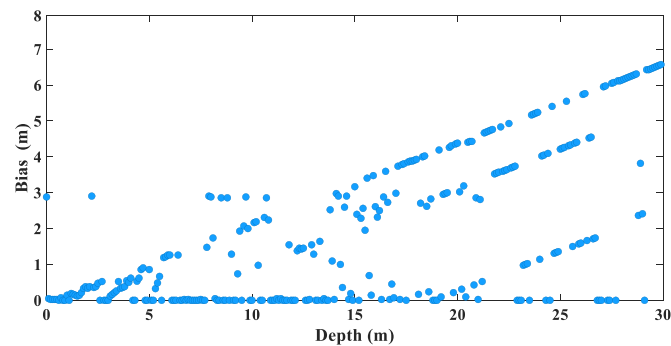

(d)

Figure 8. The influence of water depth on accuracy of water-depth extraction: (a) RLD processing, (b) BD processing, (c) RFD processing results, and (d) WFD processing results (the graphs show the absolute value of the bias).

Water depth is one of the main factors that affect the accuracy of extraction of the water depth slope distance. If the water is too shallow, the surface reflection overlaps with the bottom reflection, and the water depth cannot be extracted. If the water is too deep, the bottom reflection is masked by noise, making it impossible to extract an effective reflection signal. This is apparent from the deviation values extracted from the four groups in Figure 8: The extraction of the water depth slope distance is more accurate for water depths of 3-20 m, after which the accuracy begins to decrease. For depths greater than $25 \mathrm{~m}$, the accuracy decreases significantly. Of the four deconvolution algorithms, the RFD algorithm is the most stable against water depth interference, whereas the WFD performance is relatively unstable (WFD is most affected for the water depths of $0-5 \mathrm{~m}$ ).

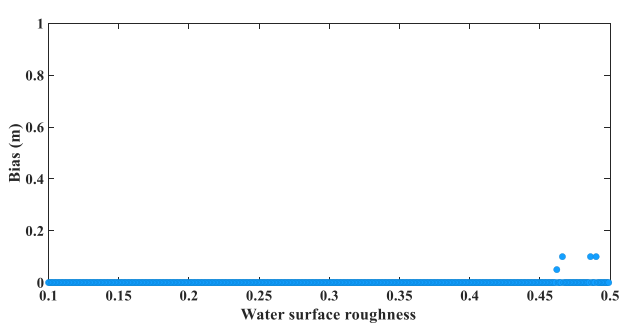

(a)

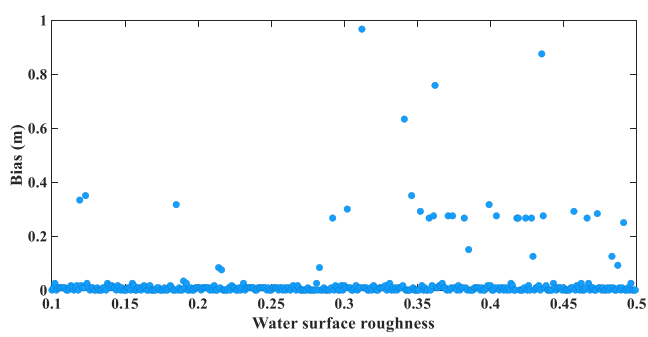

(b) 


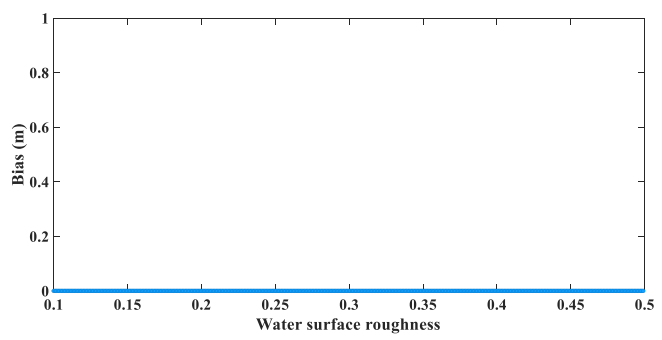

(c)

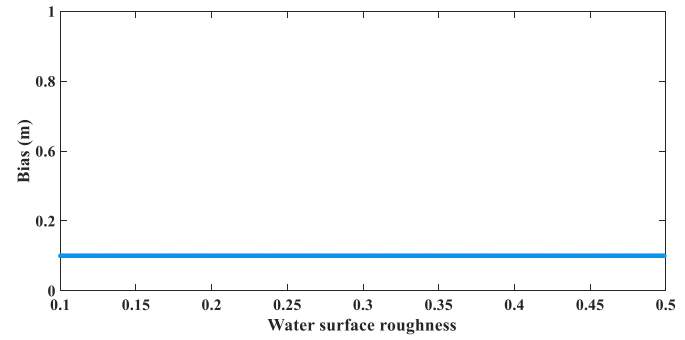

(d)

Figure 9. Effect of water-surface roughness on accuracy of water-depth extraction. (a) RLD processing, (b) BD processing, (c) RFD processing, and (d) WFD processing (the graphs show the absolute value of the bias).

Figure 9 shows that the $\mathrm{BD}$ algorithm performs poorly when considering water-surface roughness. However, compared with the influence of the other parameters, the water-surface roughness produces only a small effect on the four deconvolution algorithms. This result is attributed to the fact that the rough water surface primarily affects the water surface reflection, which is the first reflection received by the receiver, and it only transits through air, so the signal is strong. As a result, this signal is only slightly affected by water depth. However, in the process of converting the water depth slope distance to the actual water depth, the water surface roughness becomes one of the main factors affecting the actual water depth conversion because a rougher water surface leads to a greater difference between the slope distance and the actual water depth.

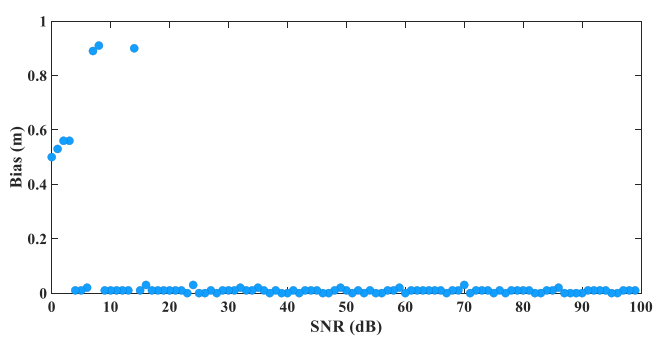

(a)

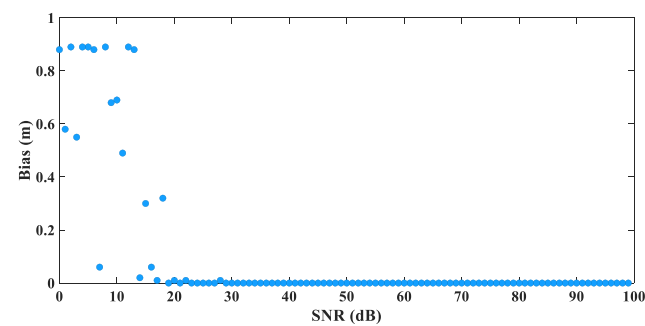

(C)

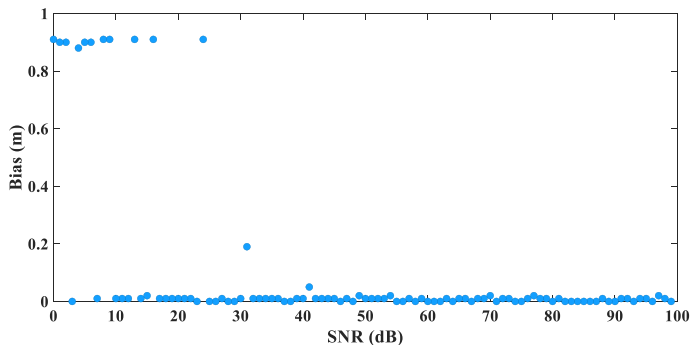

(b)

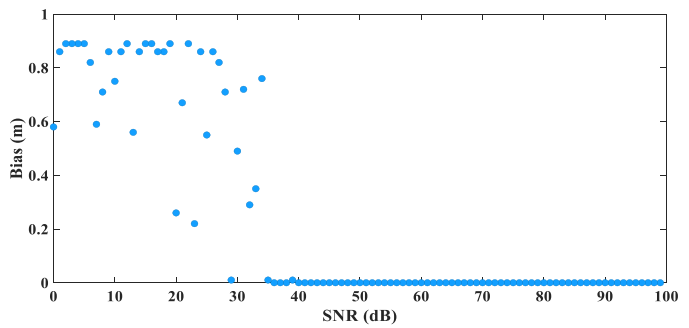

(d)

Figure 10. Influence of SNR on accuracy of water-depth extraction: (a) RLD processing, (b) BD processing, (c) RFD processing, and (d) WFD processing (the graphs show the absolute value of the bias).

Noise is also an important factor affecting water-depth extraction. When the $\mathrm{SNR}<20 \mathrm{~dB}$, the extraction accuracy of the water-depth slope varies differently for the four deconvolution algorithms (WFD is the least stable, and RLD is the most stable). When SNR $>40 \mathrm{~dB}$, the four algorithms 
perform well. Performing wavelet denoising before convolution thus improves the SNR and the accuracy of water-depth extraction.

Comparing the four deconvolution algorithms shows that RLD and BD are affected the same by convolution, and the same is true of WFD and RFD. The RLD and RFD algorithms are more robust, whereas the WFD algorithm is not. Although RFD is affected by intermittent pulses, it experiences a slight peak broadening, which is conducive to the extraction of peak time intervals. The RFD algorithm is relatively stable.

\subsection{Comparative analysis of measured data}

Before processing, the PMI1 and PMI2 channel data, the infrared channel, and the deep-water channel data are preprocessed: The reflection from the water surface is extracted from the APD data, and the bottom reflection is extracted from the PMI3 deep-water channel data. These data are used as the true values for the measured data. On the one hand, a comparison of the measured data explores the performance and difference of the deconvolution algorithm in the positive channel and the orthogonal channel and, on the other hand, tests the effectiveness of the algorithm proposed herein. Based on the characteristics of the laser reflection waveform and the simulation results, we divide the reflection data into three groups of water-depth slopes: 0-5 m, 5-25 m, and $>25 \mathrm{~m}$. Each group of data has 5000 original reflection signals. The experiments were conducted on the PMT1 and PMT2 channel data (see Tables 3-8).

Table 3. Algorithm extraction compared with measured data (water-depth slope distance is $0-5 \mathrm{~m}$, PMT1).

\begin{tabular}{ccccc}
\hline Evaluation parameter & RLD & BD & WFD & RFD \\
\hline RMSE $(\mathrm{m})$ & 0.1537 & 0.1545 & 0.3841 & 0.1945 \\
$\mathrm{R}^{2}$ & 0.9405 & 0.9435 & 0.9924 & 0.9318 \\
$\mathrm{CORR}$ & 0.8279 & 0.8293 & 0.0789 & 0.7246 \\
$\mathrm{~T}(\mathrm{~s})$ & 14.7515 & 35.8408 & 11.4099 & 14.8150 \\
\hline
\end{tabular}

Table 4. Algorithm extraction compared with measured data (water-depth slope distance is $0-5 \mathrm{~m}$,

PMT2).

\begin{tabular}{ccccc}
\hline Evaluation parameter & RLD & BD & WFD & RFD \\
\hline RMSE $(\mathrm{m})$ & 0.1537 & 0.1545 & 0.2089 & 0.1945 \\
$\mathrm{R}^{2}$ & 0.9405 & 0.9435 & 0.9143 & 0.9318 \\
CORR & 0.8279 & 0.8293 & 0.9079 & 0.7246 \\
$\mathrm{~T}(\mathrm{~s})$ & 14.7713 & 35.4752 & 14.6952 & 14.7664 \\
\hline
\end{tabular}

Tables 3 and 4 show the accuracy of shallow-water waveform extraction by using the extraction results of the four algorithms: The RLD and BD algorithms are more accurate than the WFD and RFD algorithms. Since the BD algorithm is a deconvolution operation for which the original function is assumed to be unknown, the calculation time is much longer than that of the other algorithms, and the accuracy of the BD algorithm approaches that of the RLD algorithm. The comparison of extracting via PMT1 channel data versus PMT2 channel data shows that the latter is more accurate than the former, and the accuracy of the WFD algorithm applied to PMT2 is significantly improved. A comprehensive analysis shows that the RLD algorithm works best with 
PMT2 channel data for extracting shallow water depth.

Table 5. Algorithm extraction compared with measured data (water-depth slope distance is 5-25 m, PMT1).

\begin{tabular}{ccccc}
\hline Evaluation parameter & RLD & BD & WFD & RFD \\
\hline RMSE $(\mathrm{m})$ & 0.0293 & 0.0289 & 0.2437 & 0.0420 \\
R2 & 0.9975 & 0.9985 & 0.7806 & 0.9786 \\
CORR & 0.9225 & 0.9237 & 0.0698 & 0.3206 \\
T(s) & 16.1511 & 48.9224 & 12.7001 & 15.1381 \\
\hline
\end{tabular}

Table 6. Algorithm extraction compared with measured data (water-depth slope distance is 5-25 $\mathrm{m}, \mathrm{PMT} 2)$.

\begin{tabular}{ccccc}
\hline Evaluation parameter & RLD & BD & WFD & RFD \\
\hline RMSE $(\mathrm{m})$ & 0.0279 & 0.0279 & 0.2315 & 0.0282 \\
$\mathrm{R}^{2}$ & 0.9968 & 0.9970 & 0.8258 & 0.9999 \\
CORR & 0.9150 & 0.9151 & 0.0805 & 0.8057 \\
$\mathrm{~T}(\mathrm{~s})$ & 17.6797 & 48.4024 & 12.3284 & 16.7678 \\
\hline
\end{tabular}

Tables 5 and 6 show the extraction accuracy of medium water depth, which is significantly improved compared with the accuracy of the shallow water experiment. Of the four deconvolution algorithms, the $\mathrm{BD}$ algorithm is the most accurate but has the longest calculation time. The accuracies of the RLD and RFD algorithms are similar to each other, and the correlation coefficient of RLD is better than that of RFD, but the computational efficiency of RFD is better than that of RLD. Both RLD and RFD algorithms perform well for extracting medium water depth. Comparing the precision of the extraction via PMT1 with that via PMT2 shows that PMT2 channel data are more conducive to deconvolution processing, which improves the accuracy of all four algorithms.

Table 7. Algorithm extraction compared with measured data (WFD did not detect weak bottom reflection; water-depth slope distance is $>25 \mathrm{~m}$, PMT1).

\begin{tabular}{ccccc}
\hline Evaluation parameter & RLD & BD & WFD & RFD \\
\hline RMSE $(\mathrm{m})$ & 0.2547 & 0.2561 & - & 0.4530 \\
$\mathrm{R}^{2}$ & 0.7326 & 0.7345 & - & 0.8244 \\
CORR & 0.9221 & 0.9229 & - & 0.7904 \\
$\mathrm{~T}(\mathrm{~s})$ & 19.1912 & 50.5954 & - & 17.0388 \\
\hline
\end{tabular}

Table 8. Algorithm extraction compared with measured data (WFD did not detect weak bottom reflection; water-depth slope distance is $>25 \mathrm{~m}$, PMT2).

\begin{tabular}{ccccc}
\hline Evaluation parameter & RLD & BD & WFD & RFD \\
\hline RMSE $(\mathrm{m})$ & 0.2624 & 0.2666 & - & 0.3935 \\
$\mathrm{R}^{2}$ & 0.6678 & 0.6672 & - & 0.8880 \\
CORR & 0.8960 & 0.8957 & - & 0.7598 \\
$\mathrm{~T}(\mathrm{~s})$ & 18.6591 & 50.5167 & - & 17.1501 \\
\hline
\end{tabular}

Tables 7 and 8 do not evaluate the extraction parameters of the WFD algorithm because the 
bottom reflection is weak. After deconvolution, many waveforms involve no reflection from the bottom, so the algorithm's performance in deep waters is unavailable. In deep waters, the RLD algorithm provides the most accurate deconvolution extraction, although the accuracy of the BD algorithm approaches that of the RLD algorithm. For water depth $>25 \mathrm{~m}$, the RLD and BD algorithms provide better results with PMT1 data, whereas the RFD algorithm provides better results with PMT2 data.

These results are apparent upon comparing the three groups of extraction parameters. The slope distance is most accurate for the extracted water depth of 5-25 m. The extraction accuracy decreases for a water-depth slope distance less than $5 \mathrm{~m}$ or greater than $25 \mathrm{~m}$. The measured data are consistent with the simulated results, which truly reflect how the parameters affect the algorithm. Although the WFD algorithm provides poor extraction accuracy for water depths greater than $25 \mathrm{~m}$, the other three algorithms all show provide good accuracy and robustness. The BD algorithm requires a long computation time, making it rather inefficient. The RLD algorithm processes PMT1 (PMT2) data for water depths less than (greater than) $25 \mathrm{~m}$. However, because the RLD algorithm is iterative, the RFD algorithm is more efficient. In addition, the RFD algorithm applied to the PMT2 data leads to more accurate results than when applied to the PMT1 data, so either the RLD algorithm or the RFD algorithm should be used, depending on the actual situation.

\section{Conclusion}

This paper compares the simulated results with measured data for wavelet transforms, soft threshold selection, and deconvolution. The main conclusions may be summarized as follows:

(1) For selecting wavelet bases, both db4 wavelet and Haar wavelet bases offer good noise suppression. The Haar wavelets form a rectangular wave, and that the laser emission waveform is Gaussian. To avoid introducing more errors and to accurately extract the position of the bottom reflection, the $\mathrm{db} 4$ wavelet base should be used to denoise the ALB laser echo.

(2) To select a soft threshold, the heuristic threshold denoising method is equivalent to the fixed threshold denoising method, and both are better than the extreme threshold denoising method. Based on layer-by-layer decomposition, the heuristic threshold is best for echo signal denoising, and six decomposition layers are optimal for denoising.

(3) A comparative analysis of data deconvolution before and after wavelet denoising shows that, after wavelet denoising, the WFD and RFD algorithms perform notably better for extracting reflections from the target, and the performance of RLD and BD algorithms also improves after wavelet denoising. The deconvolution experiment with the SNR model shows that a high SNR guarantees effective deconvolution. Therefore, performing a wavelet transform before the deconvolution experiment improves the deconvolution, which is particularly helpful for the RFD algorithm.

(4) The echo parameter has a varying effect on the algorithm: The RLD and RFD algorithms are more robust against such variations, whereas the WFD algorithm is less robust. Although RFD produces intermittent pulses, it has only small peak broadening, which facilitates the extraction of peak time intervals. In addition, the algorithm is stable.

(5) For multichannel data in actual experiments, both the RLD and the RFD algorithms are effective and robust. Although the accuracy of the BD algorithm is close to that of the RLD algorithm (or even superior to a certain extent), it requires a longer calculation time and thus has low computational efficiency, making it unsuitable for large-scale data 
calculation. The RFD algorithm is more suited for PMT2 data than for PMT1 data. Compared with the RLD algorithm, RFD avoids the iterative process, and the solution accuracy is close to that of RLD. Thus, RFD provides a new possibility for ALB deconvolution algorithms.

Author Contributions: Conceptualization, Y.S., H.L. and S.B.; methodology, Y.S., H.L. and G.Z.; software, Y.H.; validation, Y.S.; formal analysis, Y.S., W.Z.; investigation, Y.S., G.Z.; resources, Y.H.; writingoriginal draft preparation, Y.S.; writing-review and editing, Y.S., H.L, G.Z., Y.H. and S.B.; visualization, Y.S., W.Z.; supervision, G.Z. and S.B.; project administration, Y.S., H.L.; funding acquisition, H.L., S.B. and G.Z. All authors have read and agreed to the published version of the manuscript.

Funding: This research was funded by National Nature Science Foundation of China (No.41974005, No.41871376, No.41771487), Outstanding Youth Science Foundation of Hubei Province, China (No. 2019CFA086), and Guangxi Key Laboratory of Spatial Information and Geomatics (No. 19-050-11-02).

\section{References}

1. Ghanem, A.H.; Steffler, P.M.; Hicks, F.E.; Katopodis, C. Two dimensional hydraulic simulation of physical habitat in flowing stream. River Research and Application 1996, 12, 185-200.

2. Guenther, G.C. Digital Elevation Model Technologies and Applications: the Deem Users Manual; ASPRS: 2007; pp. pp. 237-306.

3. Tian-Yuan Shih, P.; Chen, Y.H.; Chen, J.C. Historic Shipwreck Study in Dongsha Atoll with Bathymetric LiDAR. Archaeological Prospection 2014, 21, 139-146.

4. Hochberg, E.J.; Andrefouet, S.; Tyler, M.R. Sea surface correction of high spatial resolution Ikonos images to improve bottom mapping in near-shore environments. ICES Journal of Marine Science 2003, 41, 1724-1729, doi:10.1109/TGRS.2003.815408.

5. Collin, A.; Long, B.; Archambault, P. Salt-marsh characterization, zonation assessment and mapping through a dual-wavelength LiDAR. Remote Sensing of Environment 2010, 114, 520-530.

6. Parker, H.; Sinclair, M. The successful application of Airborne LiDAR Bathymetry surveys using latest technology. In Proceedings of the Oceans, 2012.

7. Allouis, T.; Bailly, J.S.; Pastol, Y.; Roux, C.L. Comparison of LiDAR waveform processing methods for very shallow water bathymetry using Raman, near-infrared and green signals. Earth Surface Process 2010, 35, 640-650.

8. Pe'Eri, S.; Philpot, W. Increasing the existence of very shallow-water LIDAR measurements using the red-channel waveforms. IEEE Transactions on Geoscience and Remote Sensing 2007, 45, 1217-1223.

9. Wong, H.; Antoniou, A. Characterization and decomposition of waveforms for Larsen 500 airborne system. IEEE Transactions on Geoscience and Remote Sensing 1991, 29, 912 921.

10. Wong, $\mathrm{H}$.; Antoniou, A. One-dimensional signal processing techniques for airborne laser bathymetry. IEEE Transactions on Geoscience and Remote Sensing 1994, 34, 57-66.

11. Mallet, C.; Bretar, F. Full-waveform topographic lidar: State-of-the-art. Isprs Journal of Photogrammetry \& Remote Sensing 2009, 64, 1-16. 
12. Wagner W.; Ullrich A.; Melzer T.; Briese C.; Kraus K. From single-pulse to full-waveform airborne laser scanners: Potential and practical challenges. International Archives of thePhotogrammetry Remote Sensing and Spatial Information Sciences 2004.

13. Roncat A; Wagner W; Melzer T; A., U. Echo Detection and Localization in Full-Waveform Airborne Laser Scanner Data using the Averaged Square Difference Function Estimator. The photogrammetric journal of Finland 2008, 21, 62-75.

14. Wang, C.; Li, Q.; Liu, Y.; Wu, G.; Liu, P.; Ding, X. A comparison of waveform processing algorithms for single-wavelength LiDAR bathymetry. ISPRS Journal of Photogrammetry and Remote Sensing 2015, 101, 22-35.

15. Hofton, M.A.; Minster, J.B. Decomposition of laser altimeter waveforms. IEEE Transactions on Geoscience Remote Sensing of Environment 2000, 38, P.1989-1996.

16. Wagner, W.; Ullrich, A.; Ducic, V.; Melzer, T.; Studnicka, N. Gaussian decomposition and calibration of a novel small-footprint full-waveform digitising airborne laser scanner. ISPRS Journal of Photogrammetry \& Remote Sensing 2006, 60, 100-112.

17. Zhao, X.; Zhao, J.; Zhang, H.; Zhou, F. Remote Sensing of Suspended Sediment Concentrations Based on the Waveform Decomposition of Airborne LiDAR Bathymetry. Remote Sensing 2018, 10, 247.

18. Jutzi B.; Stilla U. Waveform processing of laser pulses for reconstruction of surfaces in urban areas. Measurement Techniques 2012

19. Kai, G.; Wenxue, X.; Yanxiong, L.; Xiufeng, H.; Ziwen, T. Gaussian Half-Wavelength Progressive Decomposition Method for Waveform Processing of Airborne Laser Bathymetry. Remote Sensing 2017, 10, 35.

20. Jutzi, B.; Stilla, U. Range determination with waveform recording laser systems using a Wiener Filter. Isprs Journal of Photogrammetry \& Remote Sensing 2007, 61, 95-107.

21. Wu, J.; Van Aardt, J.A.N.; Mcglinchy, J.; Asner, G.P. A Robust Signal Preprocessing Chain for Small-Footprint Waveform LiDAR. IEEE Transactions on Geoscience \& Remote Sensing 2012, 50, 3242-3255.

22. Roncat, A.; Bergauer, G.; Pfeifer, N. B-spline deconvolution for differential target crosssection determination in full-waveform laser scanning data. Isprs Journal of Photogrammetry \& Remote Sensing 2011, 66, 418-428.

23. Dong, X.; Li, G.; Jia, Y.; Li, B.; He, K. Non-iterative denoising algorithm for mechanical vibration signal using spectral graph wavelet transform and detrended fluctuation analysis. Mechanical Systems Signal Processing 149.

24. Abdallah, H. Wa-LiD: A New LiDAR Simulator for Waters. IEEE Geoscience Remote Sensing Letters 2012, 9, 744-748.

25. Parrish, C.E.; Jeong, I.; Nowak, R.D.; Smith, R.B. Empirical Comparison of Full-Waveform Lidar Algorithms. Photogrammetric Engineering and Remote Sensing 2011, 77, 825-838.

26. Bo, Z.; Sun, L.; Yu, H.; Yong, X.; Cong, Z. Wavelet denoising method for laser-induced breakdown spectroscopy. Journal of Analytical Atomic Spectrometry 2013, 28.

27. Kaufmann; Guillermo, H. Comparative study of wavelet thresholding methods for denoising electronic speckle pattern interferometry fringes. Optical Engineering 2001, 40, 2598-2604.

28. Chen, J.; Li, X.; Mohamed, M.A.; Jin, T. An Adaptive Matrix Pencil Algorithm Based-Wavelet Soft-Threshold Denoising for Analysis of Low Frequency Oscillation in Power Systems. 
IEEE Access 2020, 8, 1-1.

29. Jacob, A.M.; Menten, K.M.; Wiesemeyer, H.; Lee, M.Y.; Güsten, R.; Durán, C. Fingerprinting the effects of hyperfine structure on $\mathrm{CH}$ and $\mathrm{OH}$ far infrared spectra using Wiener filter deconvolution. Astronomy Astrophysics 2019.

30. Yeoh, W.S.; Zhang, C. Constrained Least Squares Filtering Algorithm for Ultrasound Image Deconvolution. IEEE Transactions on Biomedical Engineering 2006, 53, 2001-2007.

31. Dines; K.; Kak; A. Constrained least squares filtering. IEEE Transactions on Geoscience 1977, $25,346-350$

32. Lucy, L.B. An Iterative Technique for the Rectification of Observed Distributions. The Astronomical Journal 1974, 79, 745.

33. Cannon, M. Blind deconvolution of spatially invariant image blurs with phase. Acoustics Speech Signal Processing IEEE Transactions on 1976, 24, 58-63. 
Figures

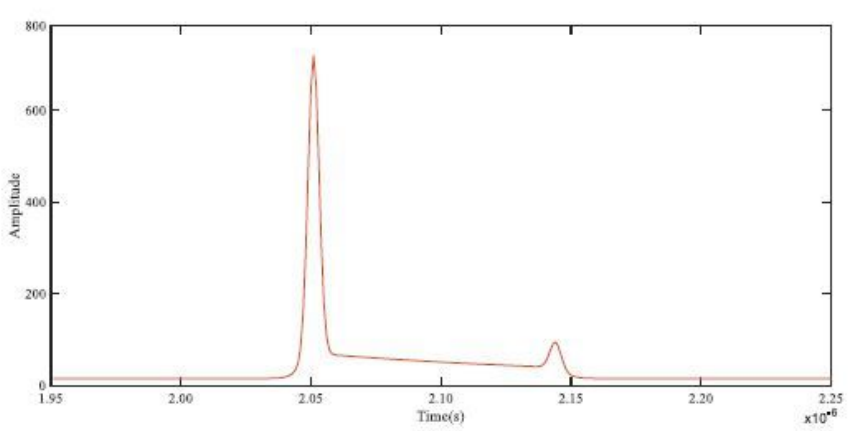

(a)

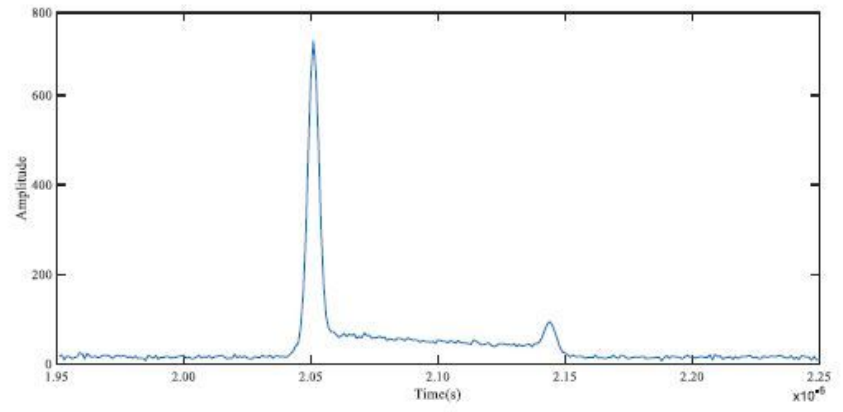

(b)

\section{Figure 1}

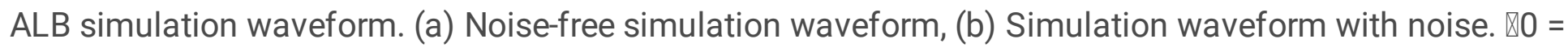

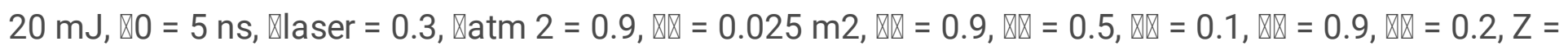

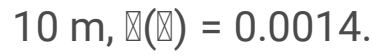




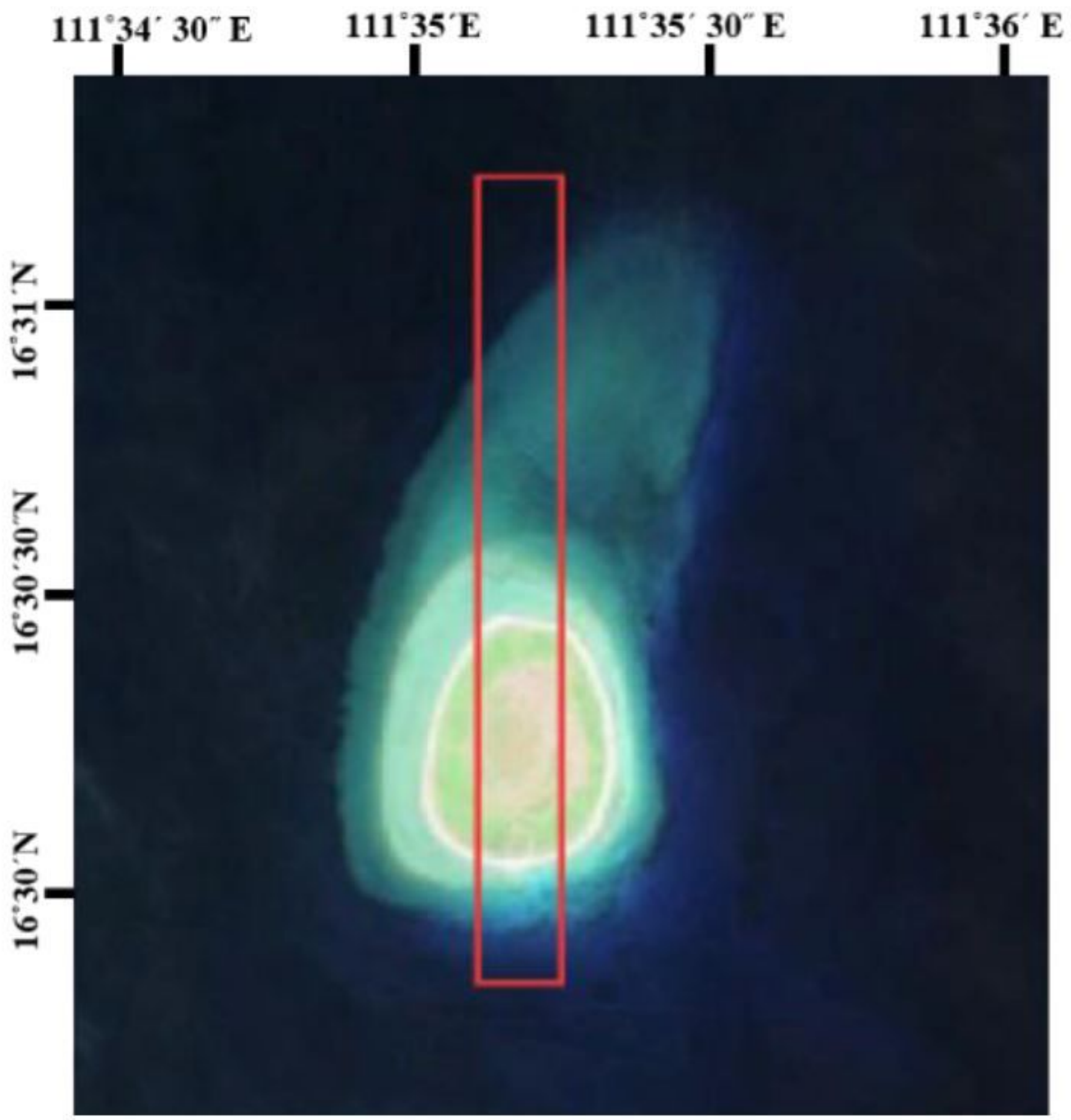

Figure 2

Study area, the red wire frame indicates the Mapper5000 aerial survey area. 


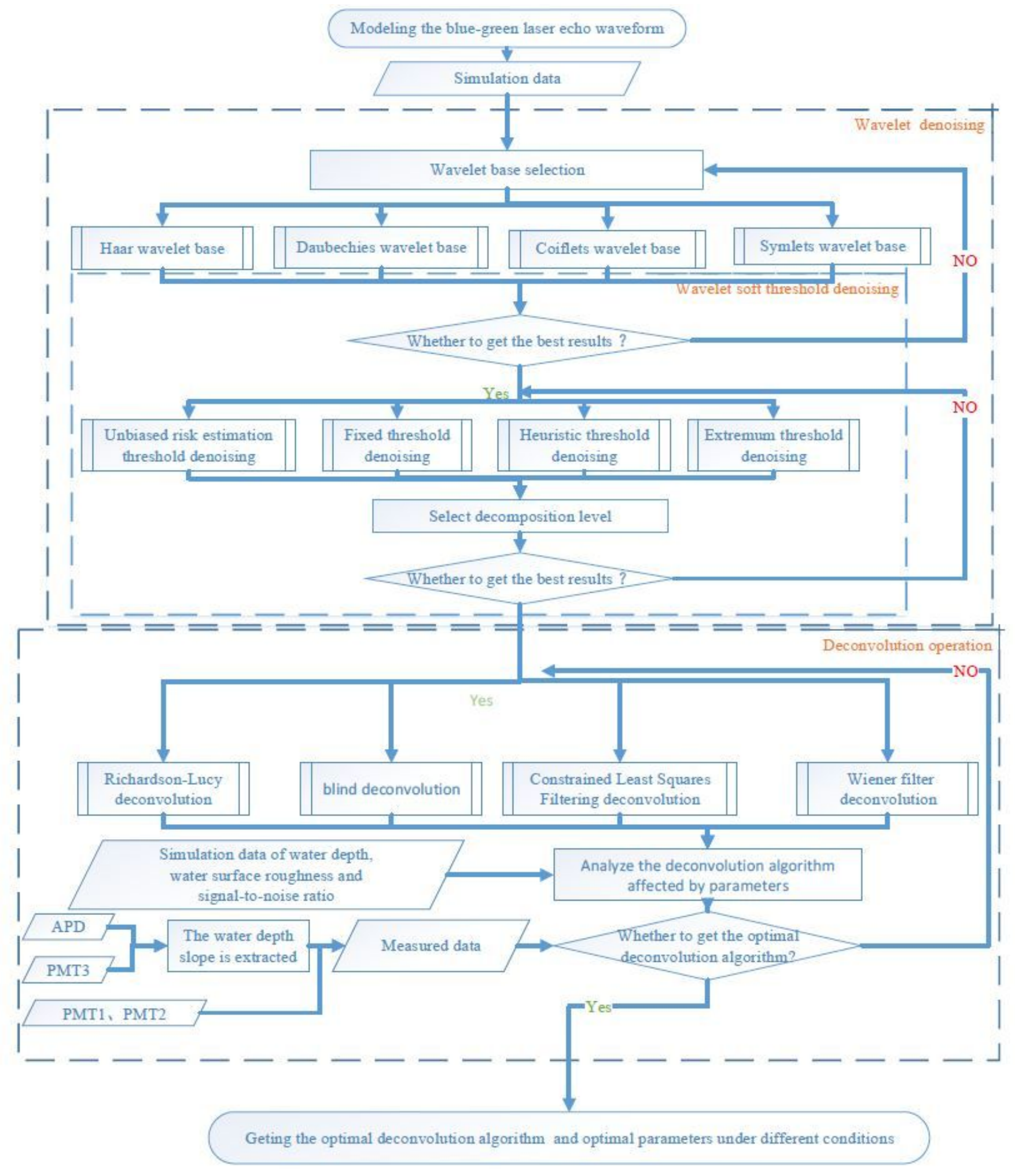

Figure 3

Flow chart of experimental process. 


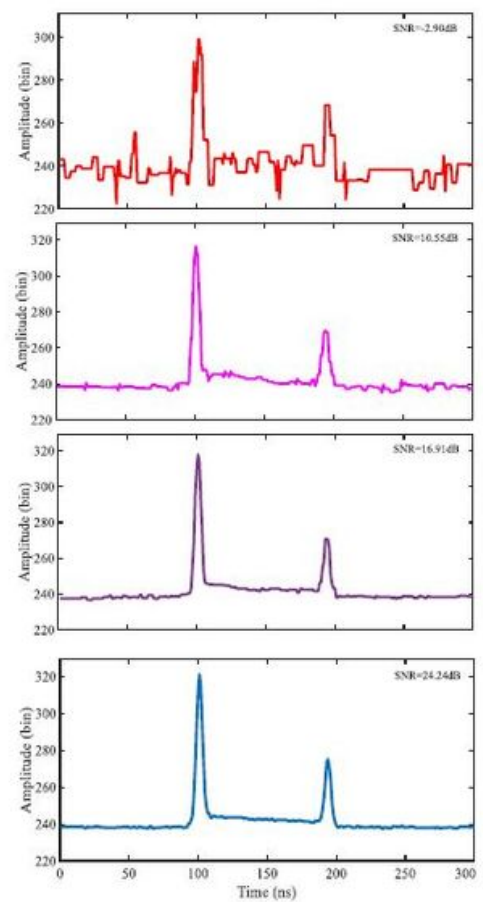

Haar wavelet denoising
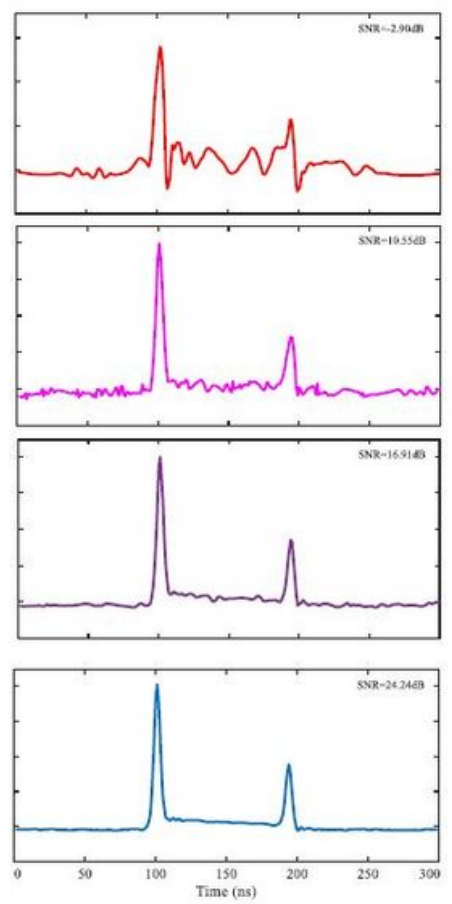

db4 wavelet denoising
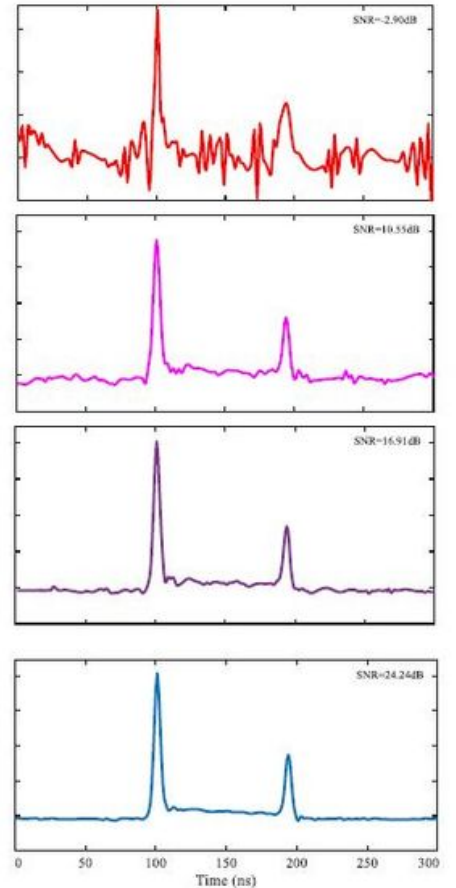

coif4 wavelet denoising
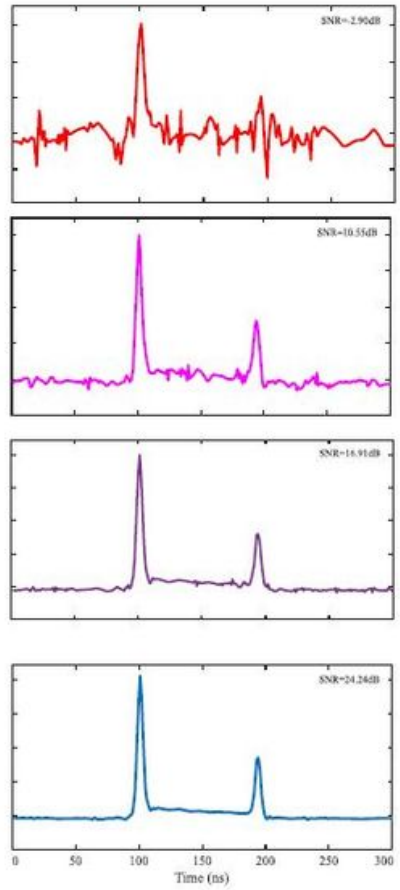

sym4 wavelet denoising

\section{Figure 4}

Effect of different wavelet basis functions on waveform denoising.

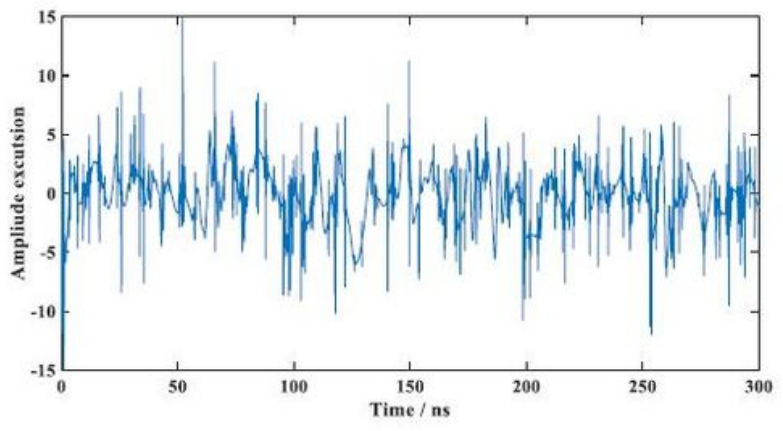

(a)

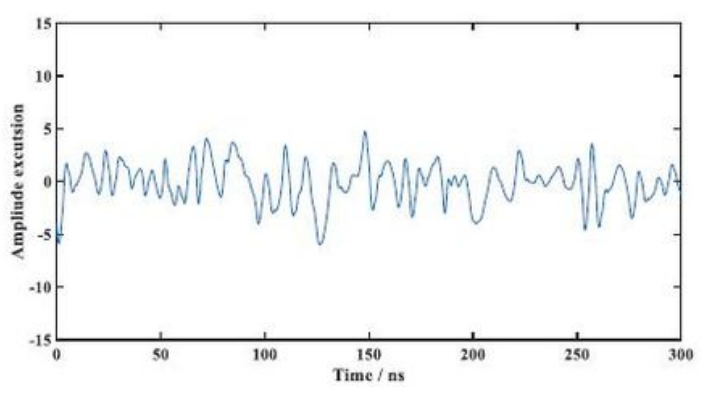

(c)

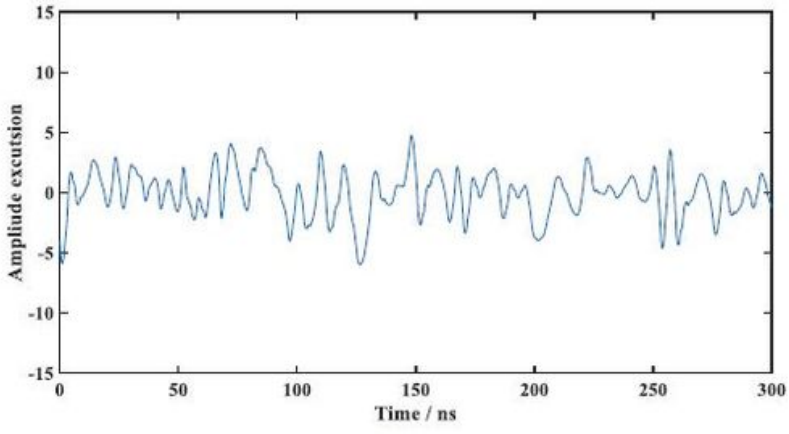

(b)

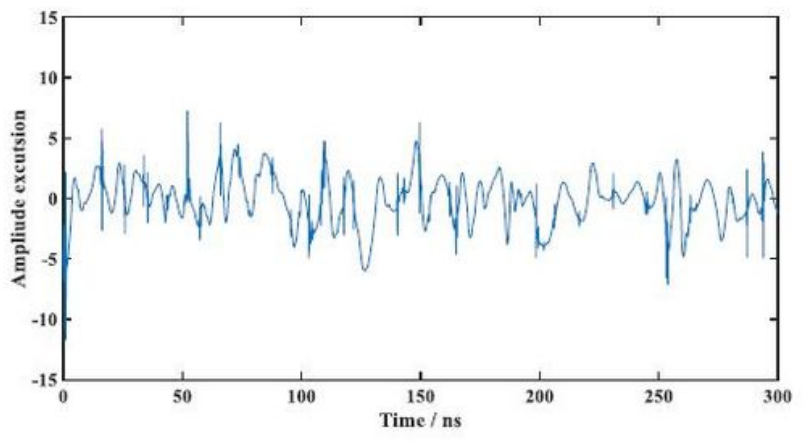

(d)

Figure 5 
Denoising deviation diagram of various thresholds: (a) unbiased risk estimation threshold denoising, (b) fixed threshold denoising, (c) heuristic threshold denoising, (d) extreme value threshold denoising.

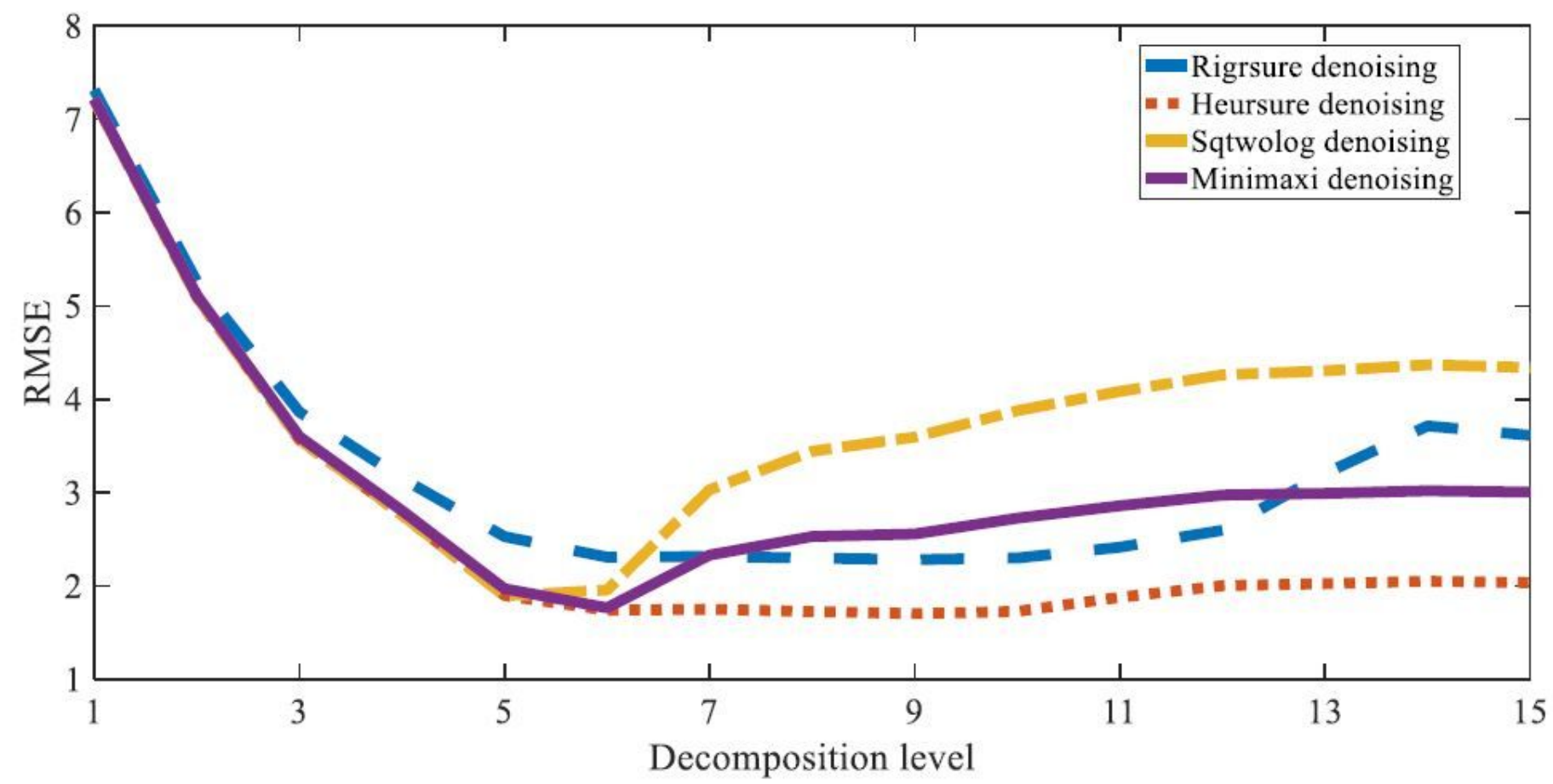

\section{Figure 6}

RMSE of denoising accuracy as a function of decomposition level to compare accuracies of wavelet threshold denoising decomposition levels.
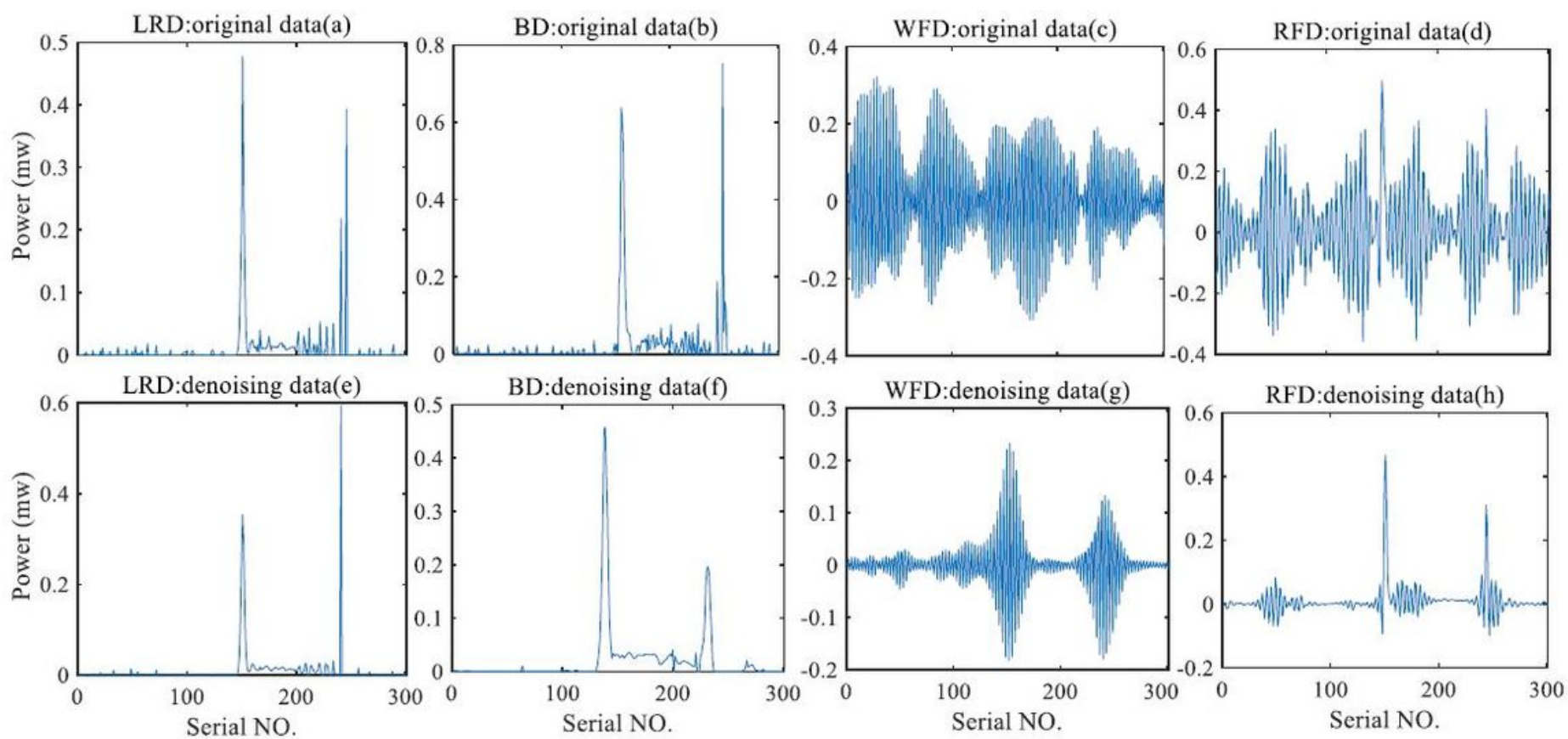

Figure 7 
Comparison of deconvolution algorithms.

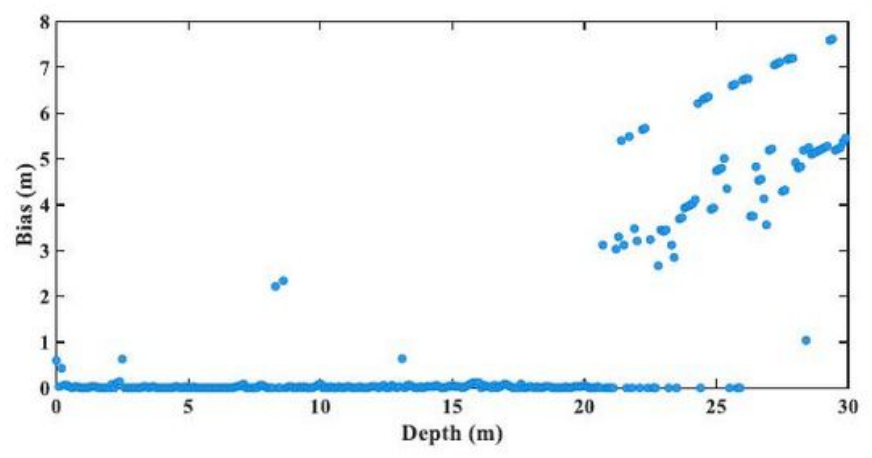

(a)

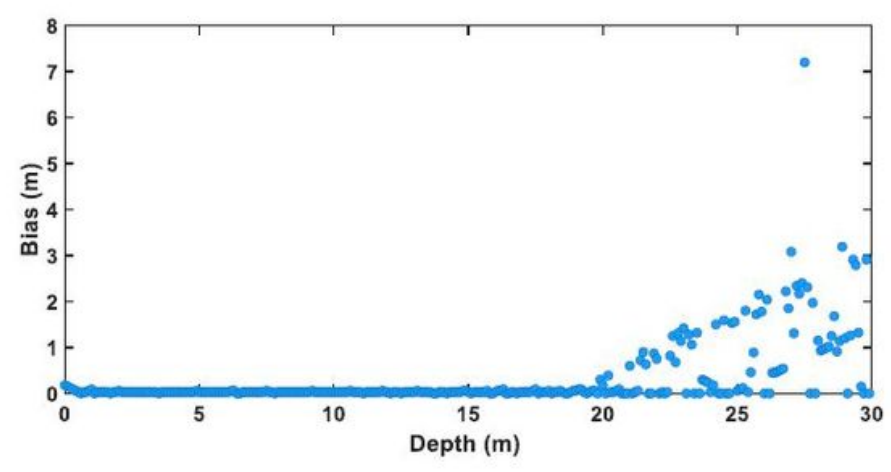

(c)

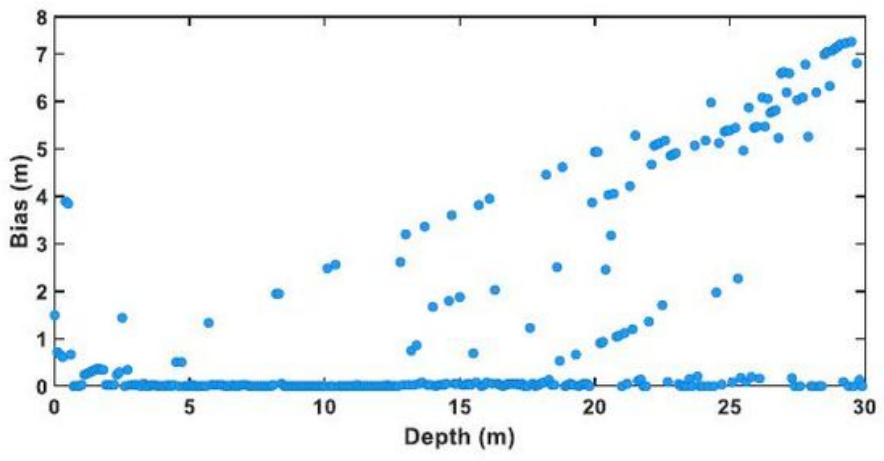

(b)

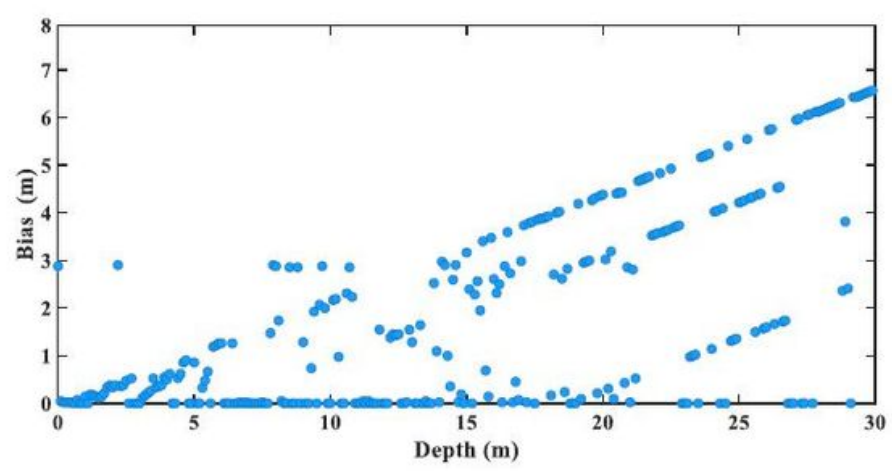

(d)

\section{Figure 8}

The influence of water depth on accuracy of water-depth extraction: (a) RLD processing, (b) BD processing, (c) RFD processing results, and (d) WFD processing results (the graphs show the absolute value of the bias). 


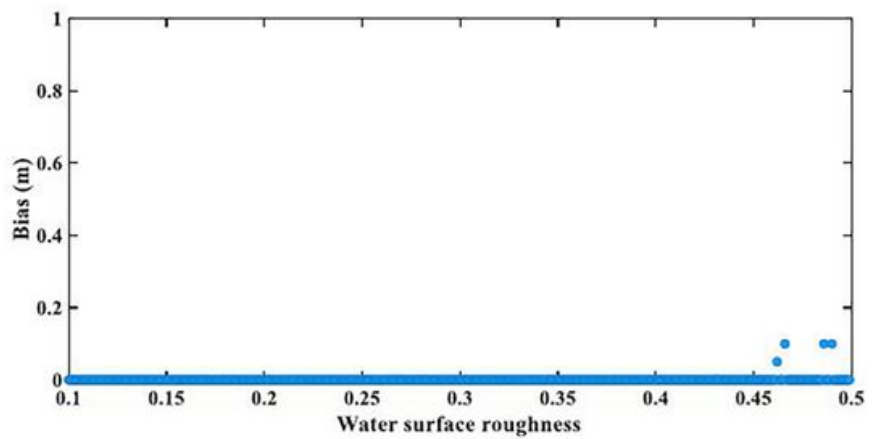

(a)

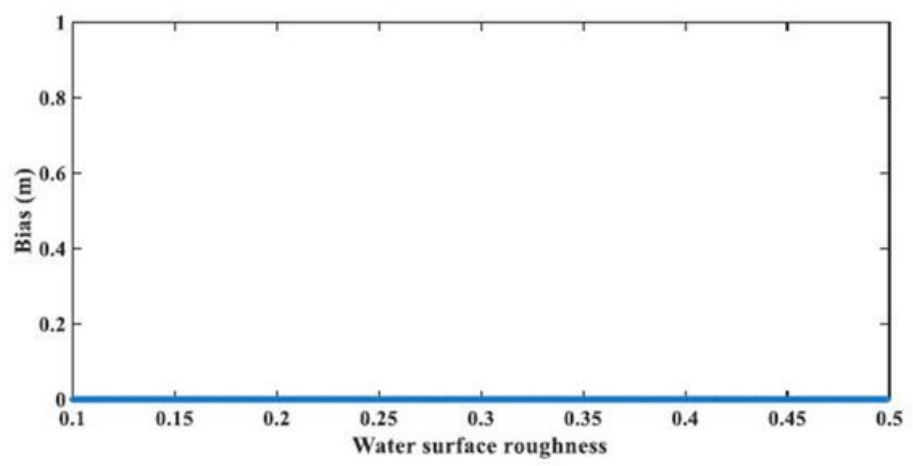

(c)

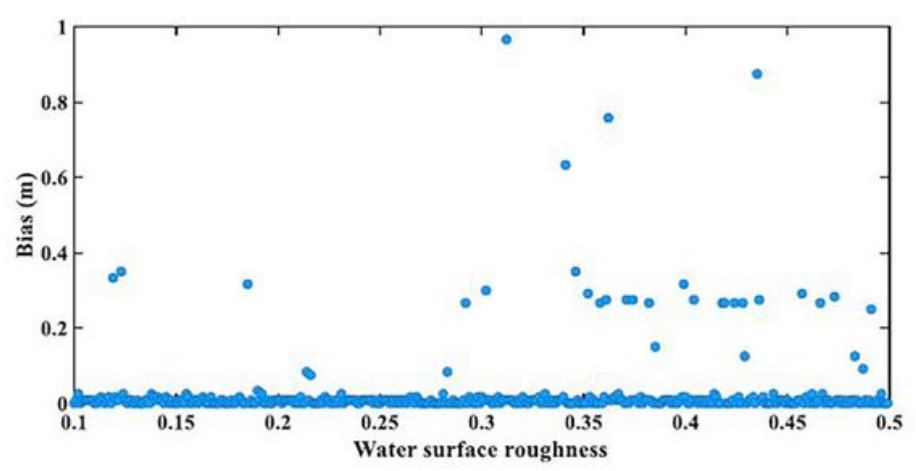

(b)

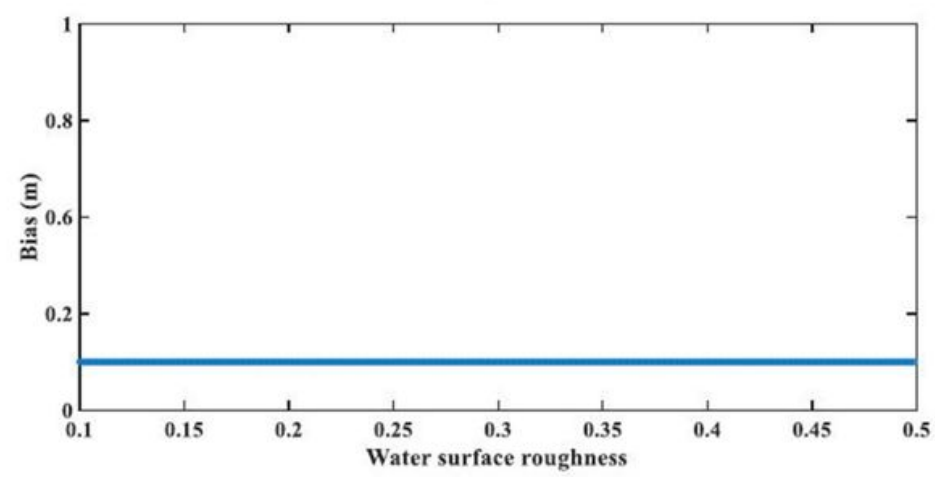

(d)

\section{Figure 9}

Effect of water-surface roughness on accuracy of water-depth extraction. (a) RLD processing, (b) BD processing, (c) RFD processing, and (d) WFD processing (the graphs show the absolute value of the bias). 


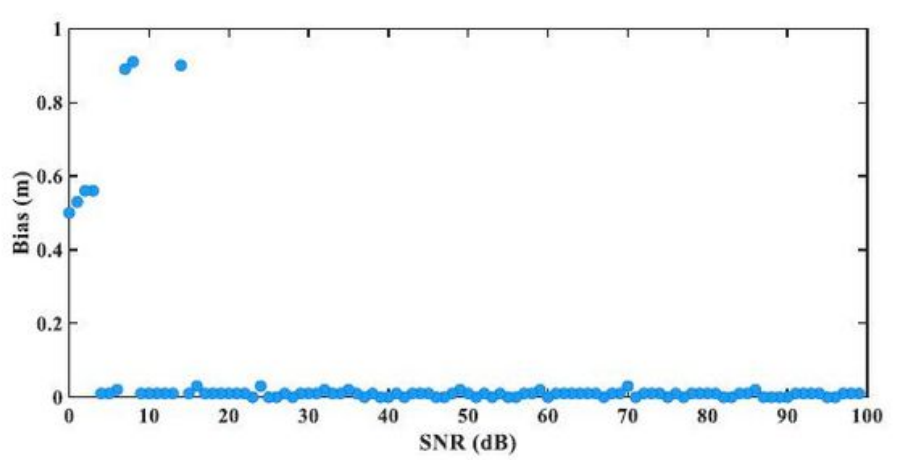

(a)

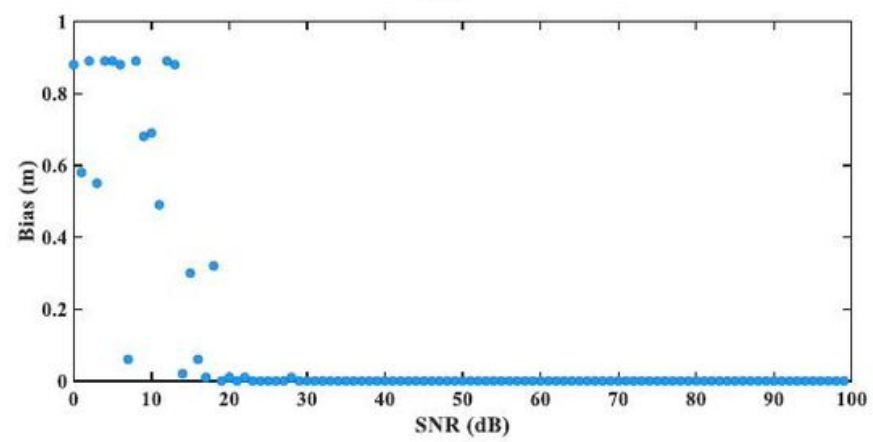

(c)

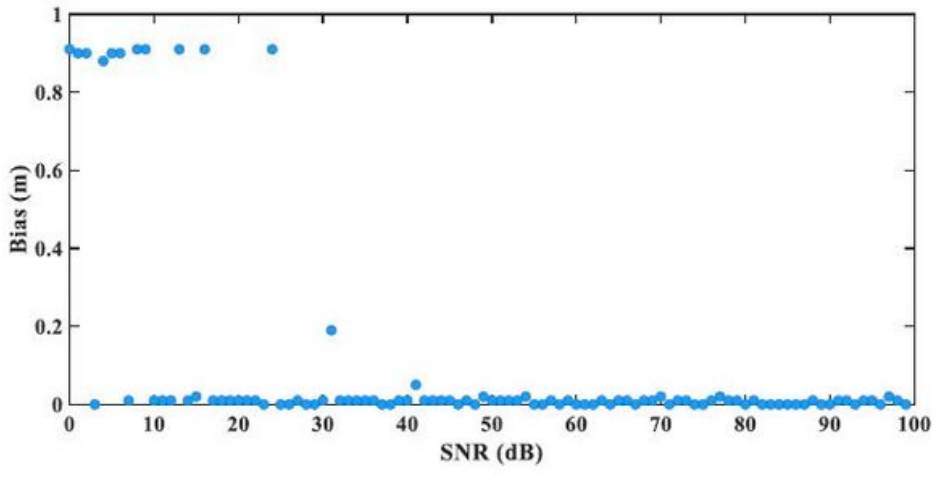

(b)

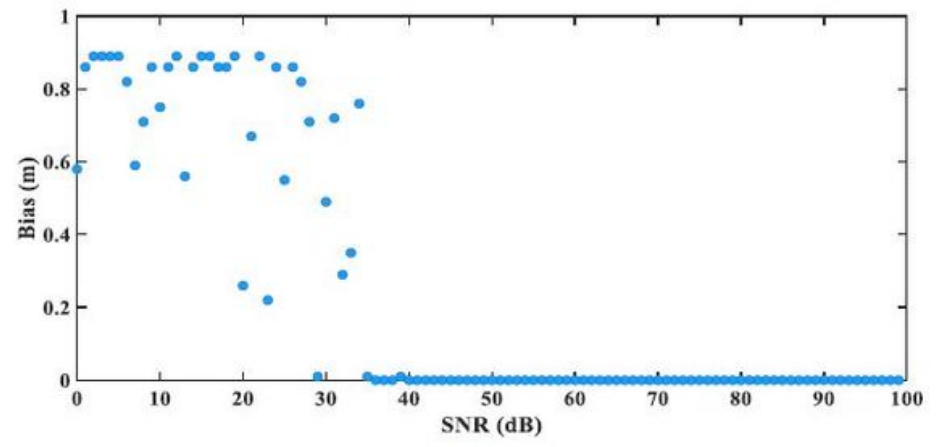

(d)

\section{Figure 10}

Influence of SNR on accuracy of water-depth extraction: (a) RLD processing, (b) BD processing, (c) RFD processing, and (d) WFD processing (the graphs show the absolute value of the bias). 